\title{
Zum Mechanismus basekatalysierter Carbodesilylierungen von Aryl- und Heteroaryltrimethylsilanen ${ }^{1)}$
}

\author{
Franz Effenberger* und Wolfgang Spiegler ${ }^{2)}$ \\ Institut für Organische Chemie der Universität Stuttgart, \\ Pfaffenwaldring 55, D-7000 Stuttgart 80
}

Eingegangen am 13. November 1984

Die Einflüsse verschiedener Basen auf die Spaltung von Silicium-Kohlenstoff-Bindungen in Aryl- und Heteroaryltrimethylsilanen unter Bildung von Aryl- bzw. Heteroaryl-Anionen in Gegenwart von Benzaldehyd als Abfangelektrophil werden untersucht. Aus den Umsetzungen mit 2-(Trimethylsilyl)benzothiazol (1) wird eine Reaktivitätsreihenfolge der verwendeten basischen Katalysatoren ermittelt. Die katalytische Aktivität der Anionen nimmt mit ihrem Ionenpotential zu. Als erster Reaktionsschritt wird der Angriff der Base am Si-Atom unter Ausbildung einer pentakoordinierten Zwischenstufe postuliert, die im geschwindigkeitsbestimmenden Schritt unter Bildung des Carbanions dissoziiert, das rasch mit dem Benzaldehyd reagiert. Die Abhängigkeit der basisch katalysierten Carbodesilylierung von der ArylAnionen-Stabilität wird an unterschiedlich substituierten Aryltrimethylsilanen 13, 13' und 13' untersucht. Die ermittelten Reaktionsgeschwindigkeiten korrelieren besser mit den Hammettschen $\sigma$-Werten als mit den Aryl-Anionen-Stabilitäten.

\section{Mechanism of Base-Catalyzed Desilylations of Aryl- and Heteroaryltrimethylsilanes ${ }^{1)}$}

The influence of different bases on the cleavage of silicium-carbon bonds in aryl- and heteroaryltrimethylsilanes is investigated in the presence of benzaldehyde as electrophilic scavenger for the aryl and heteroaryl anions formed in this process. A reactivity gradation of the various basic catalysts employed is determined from the reactions with 2-(trimethylsilyl)benzothiazole (1). The increase of catalytic activity of the anions parallels that of their ion potential. Attack of the base at the $\mathrm{Si}$ atom is postulated as the first step in this reaction sequence, with subsequent dissociation of the pentacoordinated intermediate in the ratedetermining step. The carbanion thus liberated rapidly reacts with benzaldehyde. In the differently substituted aryltrimethylsilanes 13,13 , and $13^{\prime \prime}$ the dependency of aryl anion stability in the base-catalyzed carbodesilylation was investigated. The relative rates of reaction correlate with Hammett's $\sigma$-constants rather than with the corresponding aryl anion stabilities.

Die Bedeutung der Substitution von Trimethylsilylgruppen in Aryltrimethylsilanen durch Elektrophile wurde vor allem von Eaborn et al. erkannt und untersucht ${ }^{3)}$. Unter den üblichen Bedingungen elektrophiler Aromatensubstitutionen wurde auch für die Substitution von Trimethylsilylgruppen der $\sigma$-Komplex-Mechanismus zugrundegelegt und die Bildung der kationischen Zwischenstufe (Wheland Intermediate) als geschwindigkeitsbestimmend angesehen $^{3}$.

Aufgrund der großen ipso-Geschwindigkeitsfaktoren bei elektrophilen Desilylierungsreaktionen ${ }^{4)}$ sollte eine selektive Einführung von Substituenten in Aromaten möglich sein 
und sich damit eine wichtige präparative Anwendung von Aryl- und Heteroaryltrimethylsilanen eröffnen. Eine Reihe von Arbeiten über die regioselektive Darstellung substituierter Benzole und anderer Aromaten haben diese Vorstellungen in vielfacher Weise bestätigt ${ }^{5}$.

Die in den letzten Jahren enorm angewachsene Anwendung siliciumorganischer Verbindungen in der Synthese ${ }^{6)}$ ist unter anderem darauf zurückzuführen, daß die KohlenstoffSilicium-Bindung durch Basen relativ leicht gespalten werden kann, wobei Carbanionen als reaktive Zwischenstufen entstehen, die ihrerseits weiteren Reaktionen zugänglich sind. Erwartungsgemä $B$ erfolgt die Spaltung der $\mathrm{Si}-\mathrm{C}$-Bindung um so leichter, je stabiler die gebildeten Carbanionen sind ${ }^{6}$. Da Aryl-Anionen, wie aus den Austauschgeschwindigkeiten und $\mathrm{p} K_{\mathrm{s}}$-Messungen ${ }^{7)}$ der entsprechenden Aromaten abzuleiten ist, recht instabil sind, sollte die basische Spaltung von Aryltrimethylsilanen nicht ohne weiteres erfolgen. In einer Vielzahl von Arbeiten haben hauptsächlich Eaborn et a1. ${ }^{8)}$ den Einfluß von Basen auf die Spaltung der Kohlenstoff-Silicium-Bindung untersucht und bei den Umsetzungen der Aryltrimethylsilane eine Korrelation zwischen der Spaltungsgeschwindigkeit und der Anionen-Stabilität gefunden. Da diese Reaktionen in protischen Medien durchgeführt wurden, fielen als Endprodukte ausschlieBlich die protodesilylierten Aromaten an. Versuche, diese mit Basen vollzogenen Spaltungen in Gegenwart von Elektrophilen durchzuführen, um damit zu einer präparativ interessanten Anwendung der basischen $\mathrm{Si}-\mathrm{C}$-Bindungsspaltung zu kommen, wurden nicht unternommen.

In einer Kurzmitteilung ${ }^{9}$ ' haben wir Reaktionen von Aryl- und Heteroaryltrimethylsilanen mit Elektrophilen beschrieben, die durch Zugabe katalytischer Mengen einer Base in starkem Maße beschleunigt bzw. überhaupt erst ermöglicht werden. In der vorliegenden Arbeit berichten wir über die Abhängigkeiten dieses präparativ interessanten Reaktionstyps von der Art der verwendeten Katalysatoren sowie der Struktur der Substrate.

\section{Einfluß unterschiedlicher Basen auf die Reaktivität von 2-(Trimethylsilyl)benzothiazol (1) bzw. 1-Methyl- 2-(trimethylsilyl)benzimidazol (2)}

Gilman et al. ${ }^{10)}$ haben gefunden, daß (Trimethylsilyl)pentahalogenbenzole mit Benzaldehyd unter drastischen Reaktionsbedingungen $\left(165-170^{\circ} \mathrm{C} / 4\right.$ Tage) ohne Zugabe von Katalysatoren zu Carbodesilylierungsprodukten reagieren. Bei diesen Umsetzungen scheint jedoch der normale $\sigma$-Komplex-Mechanismus wenig wahrscheinlich, zudem wurde eine starke Beschleunigung der Reaktion durch Kaliumfluorid bzw. -cyanid beobachtet ${ }^{11)}$. Dieses ungewöhnliche Reaktionsverhalten wurde durch eine primäre Addition des Nucleophils an das Silicium ( $S_{\mathrm{E}} 1(\mathrm{~N})$-Mechanismus) gedeutet ${ }^{12}$.

Systematische Untersuchungen über den EinfluB des Nucleophils auf die Spaltungsgeschwindigkeit sind uns nicht bekannt. Zumeist wurden Fluoride verwendet, wobei die große Bindungsenergie der Silicium-Fluor-Bindung ${ }^{13)}$ als entscheidend für den Reaktionsverlauf angesehen wird.

Wir haben nun am Beispiel der Umsetzungen von 2-(Trimethylsilyl)benzothiazol (1) und 1-Methyl-2-(trimethylsilyl)benzimidazol (2) mit Benzaldehyd zu den Carbodesilylierungsprodukten 3 bzw. 4 den Einfluß verschiedener Basen bzw. Nucleophile auf die Reaktionsgeschwindigkeit untersucht.

Die Reaktionen (1) wurden unkatalysiert schon durchgeführt, wobei zur Erzielung guter

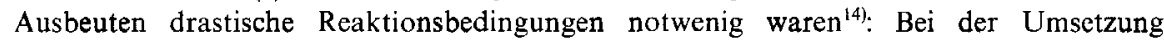
mit Benzaldehyd ergab 1 nach $40 \mathrm{~h}$ bei $160^{\circ} \mathrm{C} 78 \%$ 2-[Phenyl(trimethylsiloxy)methyl]-

Chem. Ber. 118 (1985) 
benzothiazol (3) ${ }^{14 a)}$ und 2 nach $16 \mathrm{~h}$ bei $180^{\circ} \mathrm{C} 86 \%$ 1-Methyl-2-[phenyl(trimethylsiloxy)methyl]benzimidazol (4) ${ }^{14 \mathrm{~b})}$.

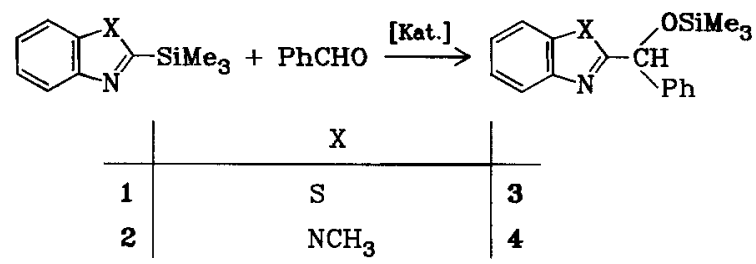

In Gegenwart von 1 mol- $\%$ Kalium-tert-butylat $\left(\mathrm{Me}_{3} \mathrm{COK}\right)$ in Dimethylformamid (DMF) erhielten wir jetzt bereits nach $15 \mathrm{~min}$ bei $-60^{\circ} \mathrm{C}$ vollständigen Umsatz von 1 zu 3. Drastischere Reaktionsbedingungen und höhere Katalysatorkonzentrationen verminderten die Ausbeuten an 3. In Gegenwart äquimolarer Mengen $\mathrm{Me}_{3} \mathrm{COK}$ entstanden z. B. unter denselben Bedingungen neben wenig 3 noch der Alkohol 5, Benzothiazol (6) sowie tert-Butyl(trimethylsilyl)ether (8) und Bis(trimethylsilyl)ether (7) (Tab. 6, exp. Teil), was auf Folgereaktionen des Carbodesilylierungsproduktes 3 mit $\mathrm{Me}_{3} \mathrm{COK}$ zurückgeführt werden konnte. So erhielten wir bei der Umsetzung von 1 mit Benzaldehyd unter portionsweiser Zugabe von $100 \mathrm{~mol}-\% \mathrm{Me}_{3} \mathrm{COK}$ nach jeder Zugabe eine Abnahme an 3 zugunsten des Alkohols 5 (Tab. 7, exp. Teil) und bei der Umsetzung von 3 mit $100 \mathrm{~mol}-\%$ $\mathrm{Me}_{3} \mathrm{COK}$ in Abwesenheit von Benzaldehyd ebenfalls den Alkohol 5. Benzothiazol (6) könnte über eine direkte Protodesilylierung des 2-(Trimethylsilyl)benzothiazols (1) entstehen, zumal bei der Umsetzung von 1 mit äquimolaren Mengen $\mathrm{Me}_{3} \mathrm{COK}$ in DMF bei $-60{ }^{\circ} \mathrm{C}$ nach $15 \min 90 \% 8$ und $20 \% 6$ entstanden und kein Ausgangsprodukt 1 mehr vorlag. Da aber bei der oben angeführten Carbodesilylierung von 1 mit Benzaldehyd in Gegenwart von $\mathrm{Me}_{3} \mathrm{COK}$ unter Feuchtigkeitsausschluß gearbeitet wurde, halten wir die Bildung von 6 über die reversible Abspaltung von Benzaldehyd aus dem Kaliumsalz von 5 für wahrscheinlicher. Aus dem in (2) formulierten Gleichgewicht kann Benzaldehyd durch irreversible Folgereaktionen (z. B. Cannizzaro-Reaktion) ausscheiden, was zu einer Verschiebung der Produktbildung von $5 \mathrm{zu} 6$ führen müßte. Da das Benzothiazolyl-Anion durch Luft-

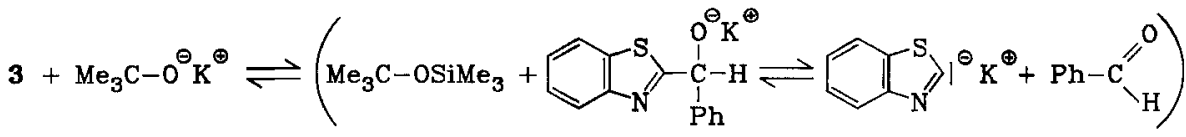

$$
\begin{aligned}
& 5 a \\
& \text { Ba } \\
& \downarrow \mathrm{H}_{2} \mathrm{O} \\
& \mathrm{Me}_{3} \mathrm{Si}-\mathrm{O}-\mathrm{SiMe}_{3}+\mathrm{Me}_{3} \mathrm{C}-\mathrm{O}-\mathrm{SiMe}_{3}+{ }_{\mathrm{N}}^{\mathrm{S}} \underset{\mathrm{P}}{\mathrm{I}}-\mathrm{H}+{ }_{\mathrm{P}}^{\mathrm{H}}+\mathrm{H} \\
& 7 \\
& 8 \\
& 5 \\
& 6
\end{aligned}
$$


Tab. 1. Carbodesilylierung von 2-(Trimethylsilyl)benzothiazol (1) mit Benzaldehyd zu 2-[Phenyl(trimethylsiloxy)methyl]benzothiazol (3) in DMF und in Gegenwart von jeweils 10 mol-\% Katalysator

\begin{tabular}{|c|c|c|c|c|c|}
\hline \multirow{2}{*}{$\frac{\text { Katalysator }^{\mathrm{a})}}{\mathrm{Me}_{3} \mathrm{COK}}$} & $\begin{array}{l}\text { Reakt.- } \\
\text { temp. }{ }^{\circ} \mathrm{C}\end{array}$ & \multicolumn{2}{|c|}{$\begin{array}{l}3 \text { Ausb. }{ }^{b)} \\
\% \text { nach () Zeit }\end{array}$} & \multicolumn{2}{|c|}{$\mathrm{p} K_{\mathrm{s}}$-Werte nach Lit. $^{18)}$} \\
\hline & -60 & $91(15 \mathrm{~min})$ & & $\mathrm{Me}_{3} \mathrm{COH}$ & 19 (Lit. $\left.{ }^{19)}\right)$ \\
\hline PhOK & -60 & $84(15 \mathrm{~min})$ & & $\mathrm{PhOH}$ & 9.89 \\
\hline $\mathrm{MeCO}_{2} \mathrm{~K}$ & $\begin{array}{r}20 \\
0\end{array}$ & $\begin{array}{l}72(15 \mathrm{~min}) \\
19(30 \mathrm{~min})\end{array}$ & $\begin{array}{r}100(25 \mathrm{~min}) \\
47(90 \mathrm{~min})\end{array}$ & $\mathrm{MeCO}_{2} \mathbf{H}$ & 4.75 \\
\hline $\mathrm{HCO}_{2} \mathrm{~K}$ & 20 & $38(2 \mathrm{~h})$ & $63(6 \mathrm{~h})$ & $\mathrm{HCO}_{2} \mathrm{H}$ & 3.75 \\
\hline $\mathrm{KF}$ & 100 & $100(1 \mathrm{~h})$ & & & \\
\hline $\mathrm{KCl}$ & 100 & $5(4 h)$ & $18(24 \mathrm{~h})$ & & \\
\hline Aliquat $336^{\mathrm{R}^{\mathrm{c}}}$ & 100 & $1(1 \mathrm{~h})$ & $13(4.5 \mathrm{~h})$ & & \\
\hline $\mathrm{Et}_{4} \mathrm{NF} \cdot 2 \mathrm{H}_{2} \mathrm{O}$ & 0 & $9(30 \mathrm{~min})$ & $23(1 \mathrm{~h})$ & & \\
\hline $\mathrm{Et}_{4} \mathrm{NBr}$ & 100 & $1(2 \mathrm{~h})$ & $4(5 \mathrm{~h})$ & & \\
\hline $\mathrm{LiF}$ & 100 & $2(19 \mathrm{~h})$ & $7(44)$ & & \\
\hline CsF & 0 & $98(30 \mathrm{~min})$ & & & \\
\hline $\mathrm{MeCO}_{2} \mathrm{Li}$ & 20 & $30(1 \mathrm{~h})$ & $49(2.25 \mathrm{~h})$ & & \\
\hline $\mathrm{MeCO}_{2} \mathrm{~K}$ & 0 & $19(30 \mathrm{~min})$ & $35(1 \mathrm{~h})$ & & \\
\hline $\mathrm{MeCO}_{2} \mathrm{NBu}_{4}$ & 0 & $44(30 \mathrm{~min})$ & $60(1 \mathrm{~h})$ & & \\
\hline$\left(\mathrm{MeCO}_{2}\right)_{2} \mathrm{Hg}$ & 20 & $2(24 \mathrm{~h})$ & $9(96 \mathrm{~h})$ & & \\
\hline
\end{tabular}

a) Zur Löslichkeit der Katalysatoren in DMF s. Tab. 8 (exp. Teil). - b) Gaschromatographisch bestimmt. - c) Fa. Fluka AG/Buchs SG: Tricaprylmethylammoniumchlorid (Gemisch von $\mathrm{C}_{8}-\mathrm{C}_{10}, \mathrm{C}_{8}$ ist vorherrschend).

Tab. 2. Vergleichende Carbodesilylierung von 2-(Trimethylsilyl)benzothiazol (1) und 1-Methyl-2-(trimethylsilyl)benzimidazol (2) mit Benzaldehyd in DMF in Gegenwart verschiedener Katalysatoren

\begin{tabular}{|c|c|c|c|c|c|c|}
\hline $\begin{array}{l}\text { Sub- } \\
\text { strat }\end{array}$ & Katalysator & mol- $\%$ & $\begin{array}{l}\text { Reakti } \\
\text { temp. C }\end{array}$ & zeit & Produkt-Ausb. ${ }^{\text {a) }}$ & $\%$ \\
\hline 1 & $\mathrm{Me}_{3} \mathrm{COK}$ & 1 & -60 & $15 \mathrm{~min}$ & $\begin{array}{l}\text { 2-[Phenyl(trimethyl- } \\
\text { siloxy)methyl]benzo- } \\
\text { thiazol (3) }\end{array}$ & $91^{\mathrm{b})}$ \\
\hline 2 & $\mathrm{Me}_{3} \mathrm{COK}$ & 1 & -60 & $15 \mathrm{~min}$ & $\begin{array}{l}\text { 1-Methyl-2-[phenyl- } \\
\text { (trimethylsiloxy)methyl]- } \\
\text { benzimidazol (4) }\end{array}$ & $98^{c)}$ \\
\hline 1 & PhOK & 10 & -60 & $15 \min$ & 3 & 84 \\
\hline 2 & PhOK & 10 & -60 & $\begin{array}{l}2 \mathrm{~h} \\
7 \mathrm{~h}\end{array}$ & $\begin{array}{l}4 \\
4\end{array}$ & $\stackrel{3}{3}^{\text {d) }}$ \\
\hline 1 & $\mathrm{MeCO}_{2} \mathbf{K}$ & 100 & 20 & $\begin{array}{l}5 \mathrm{~min} \\
10 \mathrm{~min} \\
15 \mathrm{~min} \\
25 \mathrm{~min}\end{array}$ & $\begin{array}{l}\mathbf{3} \\
\mathbf{3} \\
\mathbf{3} \\
\mathbf{3}\end{array}$ & $\begin{array}{r}23 \\
63 \\
72 \\
100\end{array}$ \\
\hline 2 & $\mathrm{MeCO}_{2} \mathrm{~K}$ & 100 & 20 & $\begin{array}{r}4 \mathrm{~h} \\
20 \mathrm{~h} \\
24 \mathrm{~h}\end{array}$ & $\begin{array}{l}4 \\
4 \\
4\end{array}$ & $\begin{array}{r}5 \\
45 \\
49\end{array}$ \\
\hline
\end{tabular}

a) Gaschromatographisch bestimmt. - ${ }^{\text {b,c) }}$ Siehe unter Carbodesilylierung von 1 und 2 mit Benzaldehyd in Gegenwart von $\mathrm{Me}_{3} \mathrm{COK} 1 \mathrm{a}$ ) und $1 \mathrm{c}$ ) (exp. Teil). - d) Nach Erwärmen auf $0{ }^{\circ} \mathrm{C}$ über Nacht $46 \% 4$.

Chem. Ber. 118 (1985) 
sauerstoff oxidativ dimerisiert (s. nachstehend), ist die Ausbeute an 6 geringer als erwartet. Eine Voraussetzung für den vorgeschlagenen Reaktionsablauf ist die große Stabilität des 2-Benzothiazolyl-Anions ${ }^{15}$.

In Tab. 1 sind die Ergebnisse der Carbodesilylierung von 1 mit Benzaldehyd in Gegenwart verschiedener Katalysatoren zusammengefaßt, wobei stets möglichst tiefe Temperaturen und kurze Reaktionszeiten angestrebt wurden, um Folgereaktionen des Carbodesilylierungsproduktes 3 mit dem Katalysator zu vermeiden. Die Aktivität der Katalysatoren kann aus den für einen vollständigen Umsatz erforderlichen Reaktionsbedingungen bzw. aus den Ausbeuten abgeleitet werden.

$\mathrm{Da}$ der Carbanionen-Stabilität am Substitutionsort entscheidende Bedeutung zukommt und sie in 2-Position des Benzothiazols (6) deutlich größer ist als in 2-Position des 1-Methylbenzimidazols (2) (die relativen Geschwindigkeiten des H/D-Austausches verhalten sich wie 350:1) ${ }^{15}$, haben wir die Carbodesilylierungsgeschwindigkeiten von 1 und 2 unter Base-Katalyse verglichen.

Tab. 2 ist zu entnehmen, daß 1 basisch katalysiert deutlich besser reagiert als 2, was sich besonders bei den weniger aktiven Katalysatoren bemerkbar macht. Bei der unkatalysierten Reaktion reagiert dagegen 2 etwas besser als $\mathbf{1}^{14)}$.

\section{Katalysatoreinfluf und Reaktionsmechanismus}

In der Literatur werden für Reaktionen von Trimethylsilanen mit Aldehyden bzw. Ketonen in Gegenwart von Basen zwei mechanistische Alternativen diskutiert.

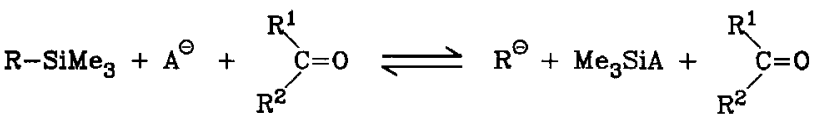

$$
\begin{aligned}
& \uparrow
\end{aligned}
$$

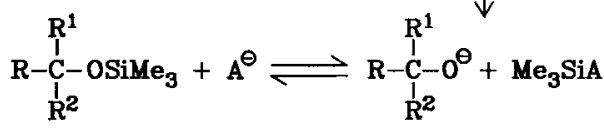

$$
\begin{aligned}
& \mathrm{R}-\mathrm{SiMe}_{3}+\mathrm{A}^{\ominus}+\underset{\mathrm{R}^{2}}{\mathrm{R}^{1} \mathrm{C}=0} \rightleftharpoons \underset{\substack{\mathrm{R}^{1} \\
\mathrm{~A}-\mathrm{C}-0^{\ominus} \\
\mathrm{R}^{2}}}{\rightleftharpoons}+\mathrm{R}-\mathrm{SiMe}_{3} \\
& \uparrow
\end{aligned}
$$

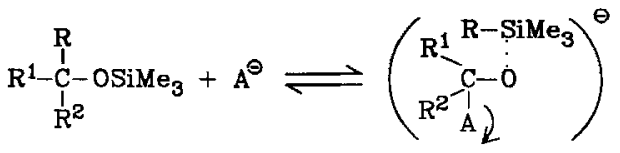

Chem. Ber. 118 (1985) 
Gleichung (3a): Addition des Anions an das Silicium unter Spaltung der $\mathrm{Si}-\mathrm{C}$ Bindung zu einem Carbanion, das sich in einer raschen Folgereaktion an die Carbonylverbindung zum entsprechenden Alkoholat addiert. Das bei der Spaltung ebenfalls entstehende $\mathrm{Me}_{3} \mathrm{SiA}$ silyliert seinerseits das Alkoholat unter Rückbildung des eingesetzten Anions ${ }^{11 b, 16)}$.

Gleichung ( 3 b): Addition des Anions an die Carbonylverbindung unter Bildung eines O-Anions, wobei in dem in der Literatur ${ }^{17)}$ angegebenen Beispiel keine $\mathrm{Si}-\mathrm{C}$-Bindung, sondern eine $\mathrm{Si}-\mathrm{H}$-Bindung gespalten wird. In den beschriebenen Beispielen ${ }^{16,17)}$ wurden Kaliumfluorid und Natrium- bzw. Kalium-alkoholate als Basen eingesetzt. Über eine abgestufte Wirksamkeit der verwendeten Anionen ist nichts angegeben.

Aus den in Tab. 1 aufgeführten Ergebnissen ergeben sich folgende Abstufungen der Katalysatorwirksamkeit bei der $\mathrm{Si}-\mathrm{C}$-Bindungsspaltung.

1. Sauerstoffnucleophile: Neben Alkoholaten sind auch Phenolate und Carboxylate zur $\mathrm{Si}-\mathrm{C}$-Spaltung geeignet. Die Reaktivitätsabstufung der jeweiligen Kaliumsalze (s. Tab. 1), $\mathrm{Me}_{3} \mathrm{COK}>\mathrm{PhOK}>\mathrm{MeCO}_{2} \mathrm{~K}>\mathrm{HCO}_{2} \mathrm{~K}$, korreliert mit den $\mathrm{p} K_{\mathrm{s}}$-Werten (Tab. 1) der zugrundeliegenden Hydroxyverbindungen, d. h. die Wirksamkeit der Anionen bei der $\mathrm{Si}-\mathrm{C}$-Spaltung steigt mit ihrer Basizität.

2. Halogenide: Grundsätzlich wäre ein Vergleich der in DMF gut löslichen Tetraalkylammoniumhalogenide am günstigsten. Da aber Tetraalkylammoniumfluoride in reiner Form äußerst instabil sind ${ }^{20)}$, haben wir einerseits die Aktivitäten von Kaliumfluorid und Kaliumchlorid in heterogener Phase und andererseits die von Aliquat $336^{\mathrm{R}}$ und Tetraethylammoniumbromid in homogener Phase verglichen (Tab. 1). Dabei zeigte sich, daß Fluoride deutlich reaktiver als Chloride und diese reaktiver als Bromide sind: $\mathrm{KF} \gg \mathrm{KCl}$; Aliquat $336^{\mathrm{R}}>\mathrm{Et}_{4} \mathrm{NBr}$.

3. Einfluß des Kations: Der Vergleich der Reaktivitätsabstufung bei Fluoriden $\mathrm{CsF} \gg \mathrm{KF} \gg \mathrm{LiF}$ und Acetaten $\mathrm{MeCO}_{2} \mathrm{NBu}_{4}>\mathrm{MeCO}_{2} \mathrm{~K}>\mathrm{MeCO}_{2} \mathrm{Li}>$ $\left(\mathrm{MeCO}_{2}\right)_{2} \mathrm{Hg}$ (Tab. 1) bestätigte die Erwartung, daß mit zunehmender Kationengröße bei gleichem Anion und Lösungsmittel durch die abnehmende Ionenpaarwechselwirkung die Anionenreaktivität größer wird ${ }^{211}$.

Bei einigen der in Tab. 1 beschriebenen Reaktionen liegt eine unterschiedliche Katalysatorkonzentration vor, da der zugegebene Katalysator nicht vollständig gelöst vorlag. Die mittels Atomabsorptionsspektrometrie bei $25^{\circ} \mathrm{C}$ bestimmte Löslichkeit der Katalysator-Salze in DMF (siehe exp. Teil, Tab. 8) zeigt jedoch, daB auch unter Einbeziehung der Löslichkeiten sich an den angegebenen Reaktivitätsabstufungen der Katalysatoren nichts Wesentliches ändert; Kaliumacetat z. B. erbrachte die beste katalytische Wirksamkeit, obwohl Kaliumformiat 16mal und Lithiumacetat sogar $150 \mathrm{mal}$ besser löslich ist als Kaliumacetat. Dagegen zeigte das in den angewandten Mengen vollständig gelöste Quecksilber(II)-acetat die kleinste katalytische Wirkung, da es in DMF praktisch undissoziiert vorliegt und daher keine Anionenaktivität aufweist. Auch Kaliumfluorid war als Katalysator wesentlich wirksamer als Kaliumchlorid, obwohl letzteres in DMF mehr als doppelt so gut löslich ist wie Kaliumfluorid (exp. Teil, Tab. 8). Bei der Diskussion des Reaktionsmechanismus scheint uns deshalb die Vernachlässigung der Katalysa- 
torlöslichkeiten gerechtfertigt. Aus unseren experimentellen Befunden schließen wir auf eine primäre Wechselwirkung des Anions mit dem Silicium. Nach Pearson $^{22)}$ ist Silicium als harte Säure einzustufen ${ }^{22 c)}$ und müßte daher mit harten Basen die energetisch günstigste Wechselwirkung eingehen. Die härteste Base ist bei den von uns eingesetzten Katalysatoren zweifellos das Fluorid-Ion, gefolgt von den Sauerstoff-Anionen. Dies erklärt auch, weshalb die bisher für $\mathrm{Si}-\mathrm{C}$-Bindungsspaltungen noch nicht verwendeten Carboxylate deutlich reaktiver sind als z. B. Chloride, obwohl die Bindungsstärken von $\mathrm{Si}-\mathrm{O}-$ bzw. $\mathrm{Si}-\mathrm{Cl}-\mathrm{Bindungen}$ vergleichbar $\operatorname{sind}^{13}$. Daß bei Reaktionen von Nucleophilen mit Siliciumverbindungen Grenzorbital-Wechselwirkungen entscheidend sind, wird in Arbeiten aus neuerer Zeit bestätigt ${ }^{23,24)}$. Im Gegensatz zu dem in (3a) vorgeschlagenen Mechanismus ${ }^{16)}$ nehmen wir die Bildung einer anionischen Zwischenstufe mit pentakoordiniertem Silicium an, die im geschwindigkeitsbestimmenden Schritt unter Bildung eines Carbanions dissoziiert, das dann, wie in ( $3 \mathrm{~b}$ ) beschrieben, weiterreagiert. In Abb. 1 ist das Reaktionsprofil, wie es sich aus unseren experimentellen Befunden ergibt, qualitativ dargestellt.

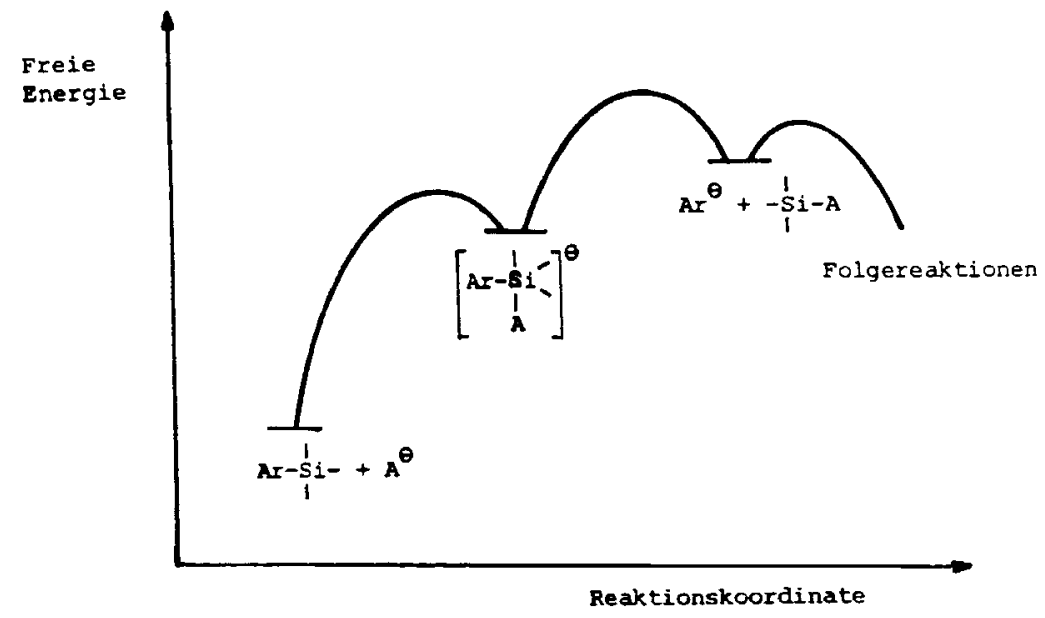

Abb. 1. Rcaktionsprofil der basekatalysierten Carbodesilylierung von Arylund Heteroaryltrimethylsilanen

Anionische pentakoordinierte Si-Verbindungen sind in der neueren Literatur beschrieben, und zwar nicht nur in Form von Reaktionszwischenstufen, sondern auch als isolierbare Verbindungen ${ }^{25}$. $\mathrm{Daß}$ bei der Umsetzung (1) von 1 mit Basen das Benzothiazolyl-Anion (6a) als Zwischenstufe auftritt, konnte durch zwei unabhängige Folgereaktionen bewiesen werden: einmal durch oxidative Dimerisierung mit Luftsauerstoff zu 2,2'-Bibenzothiazol (9) und zum zweiten durch den basekatalysierten Austausch von Trialkylsilylsubstituenten zwischen 2-(Triethylsilyl)benzothiazol (10) und 2,3,4-Trichlor-5-(trimethylsilyl)thiophen (11) zu 1 und 2,3,4Trichlor-5-(triethylsilyl)thiophen (12). 


$$
1+\mathrm{Me}_{3} \mathrm{COK} \rightarrow\left(\|\left._{N^{-S}}^{\mathrm{S}}\right|^{\ominus}{ }^{\oplus}\right)+\mathrm{Me}_{3} \mathrm{C}-0-\mathrm{SiMe}_{3}
$$

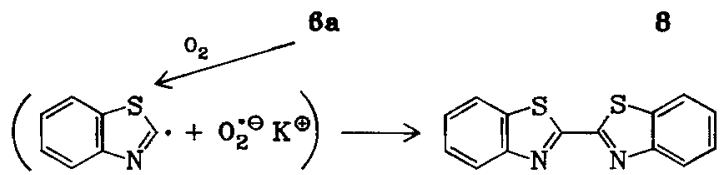

$6 \mathrm{~b}$

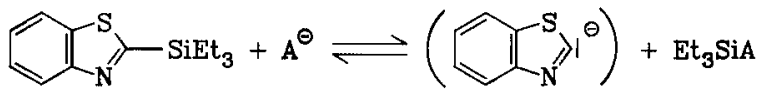

10

6a
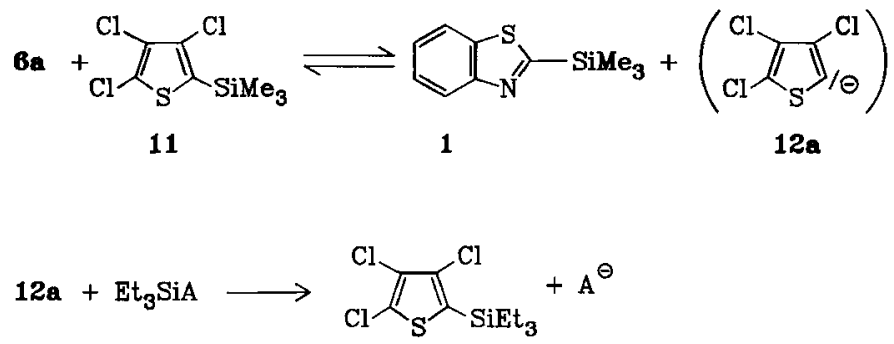

Die Möglichkeit einer Unterstützung der Dissoziation der anionischen pentakoordinierten Silicium-Zwischenstufe (Abb. 1) durch Elektrophile kann im Prinzip als Erhöhung der Austrittstendenz des entsprechenden Carbanions angesehen werden ${ }^{23)}$ und wird um so wichtiger, je instabiler dieses Carbanion ist. Der Reaktivitätsunterschied von 1 und 2 (Tab. 2) läßt sich anhand des Reaktionsprofils (Abb. 1) zwanglos deuten. Bei vergleichbarer Stabilität der pentakoordinierten Silicium-Zwischenstufen von 1 und $\mathbf{2}$ ist die Dissoziation zum schlechter stabilisierten Benzimidazol-Anion ${ }^{15)}$ erschwert, und die Reaktion läuft erwartungsgemäß langsamer ab. Mit abnehmender Anionenstabilität und zunehmender Basizität des Heterocyclus kann jedoch auch die Adduktbildung zwischen dem zweifach koordinierten Stickstoff im Heterocyclus und Benzaldehyd und dessen Folgereaktion mit dem anionischen Katalysator entscheidend werden - ein Mechanismus, wie er für Protodesilylierungen bei Heteroarylsilanen diskutiert wird ${ }^{26)}$ - , worauf jedoch in dieser Arbeit nicht näher eingegangen werden soll. Abb. 1 ist weiter zu entnehmen, daß auch die Stabilität der gebildeten Si-A-Bindung die Reaktionsgeschwindigkeit beeinflussen wird; je stabiler diese Bindung, um so niedriger wird die Aktivierungsenergie für den entscheidenden Dissoziationsschritt. Es wäre jedoch falsch, allein aus den Bindungsenergien ${ }^{13)}$ (thermodynamische Kontrolle) die Möglichkeit der Spaltung von Si-C-Bindungen mit Anionen ableiten zu wollen. 
Der unterschiedliche Einfluß der verschiedenen Basen in den untersuchten Reaktionen hat eindeutig gezeigt, daß die Bildung und Stabilität von Reaktionszwischenstufen und damit die Energie der Übergangszustände (kinetische Kontrolle) einen dominierenden Einfluß auf diese Spaltungsreaktionen hat.

\section{Basekatalysierte Reaktionen von Aryltrimethylsilanen mit Benz- aldehyd - Substituentenabhängigkeit}

Nach dem außergewöhnlich großen Einfluß basischer Katalysatoren, den wir bei den Umsetzungen der Heteroaryltrimethylsilane 1 und 2 mit Benzaldehyd festgestellt haben, erschien es möglich, daß außer den (Pentahalogenphenyl)trimethylsilanen ${ }^{10,11)}$ auch andere substituierte Aryltrimethylsilane mit Benzaldehyd in Gegenwart basischer Katalysatoren unter Carbodesilylierung reagieren. Reaktionen dieses Typs wären präparativ von großem Interesse, da sie die elektrophile Einführung von Substituenten besonders in Acceptor-substituierte Aromaten ermöglichen würden. Wir haben deshalb eine Reihe monosubstituierter Phenyltrimethylsilane untersucht und solche Verbindungen ausgewählt, die aufgrund ihrer Substituenten Aryl-Anionen in abgestufter Form zu stabilisieren vermögen. Da die Stabilisierung der Anionen auch von der Position der Substituenten im Ring abhängt, haben wir auch Stellungsisomere untersucht.

Für Protodesilylierungen von Aryltrimethylsilanen im basischen Medium wurden eindeutige Abhängigkeiten der Reaktionsgeschwindigkeiten von den Substituenten im Phenylring gefunden ${ }^{81}$, wobei die Geschwindigkeiten einerseits mit den Aryl-Anionen-Stabilitä$\operatorname{ten}^{8 \mathrm{~b}, \mathrm{c})}$, andererseits mit den Hammettschen $\sigma$-Konstanten ${ }^{8 \mathrm{c}, \mathrm{d})}$ korrelieren. Ähnlich abgestufte Reaktionsgeschwindigkeiten sollten auch für die basisch katalysierten Carbodesilylierungen zu erwarten sein.

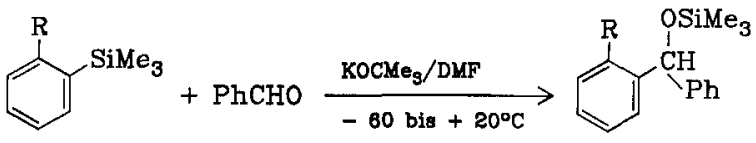

13

14

\begin{tabular}{l|lllll} 
& $\mathbf{a}$ & $\mathbf{b}$ & $\mathbf{c}$ & $\mathbf{d}$ & $\mathbf{e}$ \\
\hline $\mathrm{R}$ & $\mathrm{NO}_{2}$ & $\mathrm{Cl}$ & $\mathrm{F}$ & $\mathrm{C}_{6} \mathrm{H}_{5} \mathrm{O}$ & $\mathrm{C}_{6} \mathrm{H}_{5} \mathrm{SO}_{2}$
\end{tabular}

Zunächst setzten wir die in Tab. 3 aufgeführten 2-substituierten (Trimethylsilyl)benzole 13 mit Benzaldehyd unter Basekatalyse um; die 2-Nitro-, 2-Chlor-, 2-Fluor-, 2-Phenoxy- und 2-(Phenylsulfonyl)-Derivate 13a-e lieferten mit $\mathrm{Me}_{3} \mathrm{COK}$ als Katalysator in DMF bei $-60^{\circ} \mathrm{C}$ bis Raumtemperatur rasch die Trimethylsilylether $14 \mathrm{a}-\mathrm{e}$ in guten Ausbeuten.

Die 2-Brom-, 2-Methoxy-, 2-(Ethoxycarbonyl)- und 2-Benzoyl-Derivate 13f-i reagierten mit Benzaldehyd nur in Hexamethylphosphorsäuretriamid (HMPT) mit Cäsium- bzw. Kaliumfluorid bei $100^{\circ} \mathrm{C}$ langsam, wobei z. T. Folgeprodukte der erwarteten Carbodesilylierungsprodukte 14 bzw. 15 isoliert wurden (Tab. 3).

2-Brom-1 -(trimethylsilyl)benzol (13f) zeigte einen unerwartet hohen Reaktionsabfall zur entsprechenden Chlorverbindung 13b. Die Umsetzung von 2-Methoxy1-(trimethylsilyl)benzol (13g) führte nach hydrolytischer Aufarbeitung zu einem 


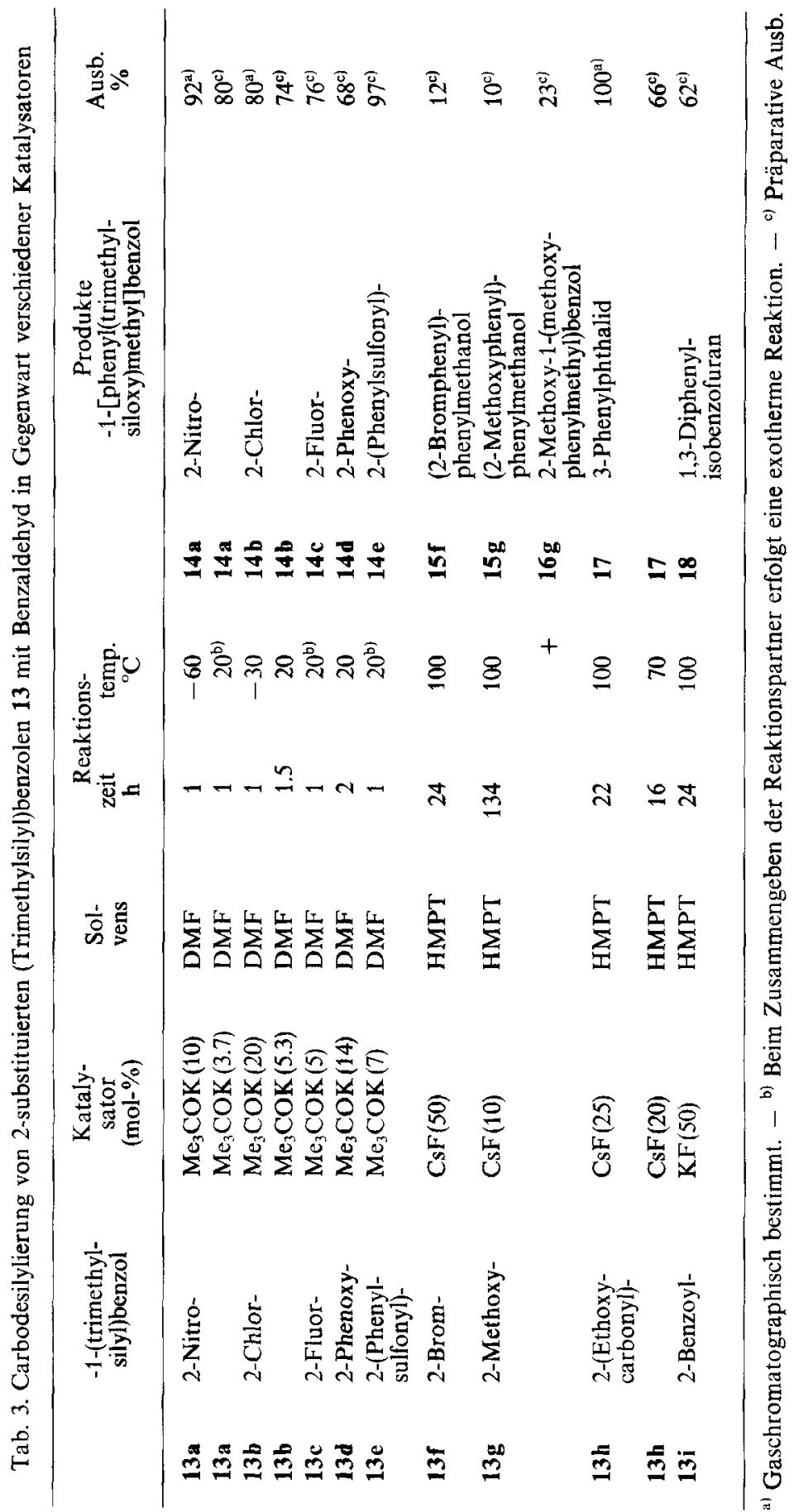


Gemisch aus Silylether $14 \mathrm{~g}$ und Alkohol 15g. Bei der Umsetzung des Gemisches mit wäßriger Salzsäure erfolgte teilweise Veretherung zu 2-Methoxy-1-(methoxyphenylmethyl)benzol (16g).

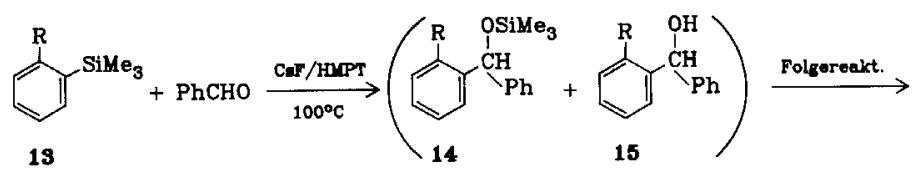

13

\begin{tabular}{c|lllll}
$13,14,15$ & I & \& & h & i \\
\hline $\mathrm{R}$ & $\mathrm{Br}$ & $\mathrm{MeO}$ & $\mathrm{CO}_{2} \mathrm{Et}$ & $\mathrm{COPh}$
\end{tabular}
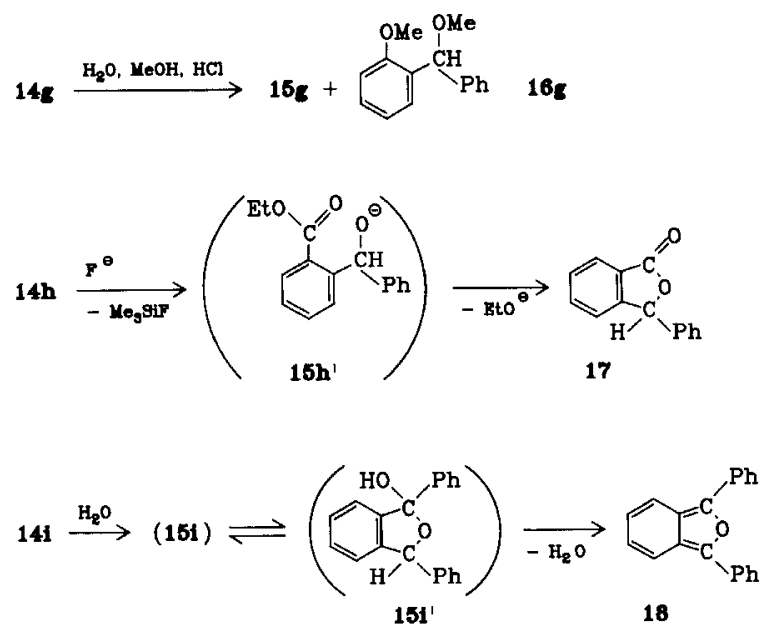

Die Versuche mit 2-(Ethoxycarbonyl)-1-(trimethylsilyl)benzol (13h) führten nach Aufarbeiten mit wäßrigem Methanol und verdünnter Salzsäure zu 3-Phenylphthalid (17). Eine intermediäre Bildung des erwarteten Trimethylsilylethers 14h oder des entsprechenden Alkohols $15 \mathrm{~h}$ konnte nicht nachgewiesen werden, so daß wir eine Reaktion über das Alkoholat $\mathbf{1 5} \mathbf{h}^{\prime}$ unter Lactonbildung annehmen.

Aus 2-Benzoyl-1-(trimethylsilyl)benzol (13i) erhielten wir nach wäßriger Aufarbeitung und Erhitzen des erhaltenen Produkts auf $100^{\circ} \mathrm{C}$ i. Vak. 1,3-Diphenylisobenzofuran (18), dessen Bildung wir über die Carbodesilylierung von 13i zum Silylether 14i, dessen Hydrolyse zum Alkohol 15i und nachfolgende Dehydratisierung des Halbacetals $15 i^{27)}$ erklären.

$\mathrm{Da} \beta$ die meisten der bei der Umsetzung des 2-(Trimethylsilyl)benzothiazols (1) wirksamen Katalysatoren (s. Tab. 1) prinzipiell auch für die Carbodesilylierung der Aryltrimethylsilane 13 geeignet sind, haben wir am Beispiel der Umsetzung von 13a mit verschiedenen Katalysatoren gezeigt. Aus den für eine vollständige Umsetzung erforderlichen Bedingungen sowie den hierbei erzielten Ausbeuten (s. Tab. 4) lassen sich wiederum die unterschiedlichen Katalysatoraktivitäten ablesen. Auch hier erwiesen sich $\mathrm{Me}_{3} \mathrm{COK}$ und Tetramethylammoniumfluorid als die wirksamsten Katalysatoren, gefolgt von Cäsiumfluorid. Für eine erfolgreiche Car- 
bodesilylierung mit Kaliumfluorid und Tetrabutylammoniumacetat waren dagegen $80^{\circ} \mathrm{C}$, mit Kaliumacetat $100^{\circ} \mathrm{C}$ erforderlich.

Tab. 4. Carbodesilylierung von 2-Nitro-1-(trimethylsilyl)benzol (13a) mit Benzaldehyd zu 2-Nitro-1-[phenyl(trimethylsiloxy)methyl]benzol (14a) bzw. (2-Nitrophenyl)phenylmethanol (15a) in DMF und in Gegenwart von jeweils $10 \mathrm{~mol}-\%$ Katalysator

\begin{tabular}{|c|c|c|c|c|}
\hline \multirow{2}{*}{ Katalysator ${ }^{a)}$} & \multicolumn{2}{|c|}{$\begin{array}{l}\text { Reaktionsbedingungen } \\
\text { für } 100 \% \text { Umsatz }\end{array}$} & \multicolumn{2}{|c|}{$\underset{\%}{\text { Ausb. }}$} \\
\hline & $\underset{{ }^{\circ} \mathrm{C}}{\text { Temp. }}$ & $\underset{\mathrm{h}}{\text { Zeit }}$ & $14 \mathrm{a}$ & $15 \mathbf{a}$ \\
\hline $\mathrm{Me}_{3} \mathrm{COK}$ & -60 & 1 & 92 & \\
\hline $\mathrm{MeCO}_{2} \mathrm{~K}$ & 100 & 6 & 45 & \\
\hline KF & 80 & 1 & 91 & \\
\hline $\mathrm{CsF}$ & 20 & 2 & 32 & $66^{c)}$ \\
\hline $\mathrm{Et}_{4} \mathrm{NF} \cdot 2 \mathrm{H}_{2} \mathrm{O}$ & 20 & $1 / 4$ & 73 & \\
\hline $\mathrm{MeCO}_{2} \mathrm{NBu}_{4}$ & 80 & 18 & 54 & \\
\hline
\end{tabular}

a) Zur Löslichkeit der Katalysatoren in DMF s. Tab. 8 (exp. Teil). - ${ }^{\text {b) }}$ Gaschromatographisch bestimmt. - ${ }^{\text {c) }}$ Durch Hydrolyse aus 14a bei wäßriger Aufarbeitung entstanden.

Die in 3- bzw. 4-Stellung Nitro- und Chlor-substituierten (Trimethylsilyl)benzole $13^{\prime} \mathbf{a}, 13^{\prime \prime} \mathbf{a}$ und $13^{\prime} \mathbf{b}, 13^{\prime \prime} \mathbf{b}$ erforderten erwartungsgemäß drastischere Reaktionsbedingungen bei der basekatalysierten Carbodesilylierung mit Benzaldehyd als die entsprechenden 2-Isomeren 13a, b (s. exp. Teil).
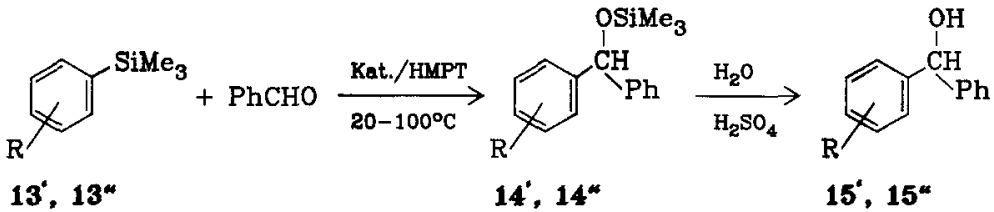

\begin{tabular}{|c|c|c|c|c|c|c|c|}
\hline & $13^{\prime}$ a $14^{\prime}$ a $15^{\circ}$ a & $13^{\mu} a$ & $14^{\prime \prime} \mathrm{a} \quad 15^{\mu} \mathrm{a}$ & $13^{\prime} b$ & $14^{\prime} \mathrm{b} \quad 15^{t} \mathrm{~b}$ & $13^{\omega} \mathrm{b}$ & $14^{\prime \prime b}$ \\
\hline $\mathrm{R}$ & $3-\mathrm{NO}_{2}$ & & $4-\mathrm{NO}_{2}$ & & $3-\mathrm{Cl}$ & & $4-\mathrm{Cl}$ \\
\hline
\end{tabular}

Als Lösungsmittel wählten wir hierbei HMPT, da sich der 4-Nitro-substituierte Trimethylsilylether 14"a mit $\mathrm{Me}_{3} \mathrm{COK}$ in HMPT langsamer als in DMF zu 1-Benzoyl-4-nitrobenzol (20"a) oxidiert (s. exp. Teil).

Bei der Verfolgung des Reaktionsablaufs der Carbodesilylierung von 3-Nitro1-(trimethylsilyl)benzol (13'a) unter portionsweiser Zugabe von insgesamt $20 \mathrm{~mol}$ $\% \mathrm{Me}_{3} \mathrm{COK}$, jeweiligem Nachrühren und gaschromatographischer Bestimmung der Produktverteilung beobachteten wir mit zunehmender Reaktionsdauer eine Abnahme des Primärprodukts 3-Nitro-1-[phenyl(trimethylsiloxy)methyl]benzol (14'a) zugunsten der Bildung von (3-Nitrophenyl)phenylmethanol (15'a) und 1-Benzoyl-3-nitrobenzol (20'a), letzteres wurde nach 14tägigem Stehenlassen bei

Chem. Ber. 118 (1985) 
Raumtemperatur und wäßriger Aufarbeitung als einziges Produkt mit 20\% Ausbeute isoliert. Wir nehmen daher infolge der geringeren Reaktivität des 3-Nitro(13'a) im Vergleich zu der des 2-Nitro-1-(trimethylsilyl)benzols (13a) eine Konkurrenzreaktion des gebildeten Trimethylsilylethers 14'a mit dem Katalysator zum sekundären Alkoholat 19'a an, das durch Luftsauerstoff oder durch die Nitroverbindung selbst weiter zu $20^{\prime}$ a oxidiert wird.

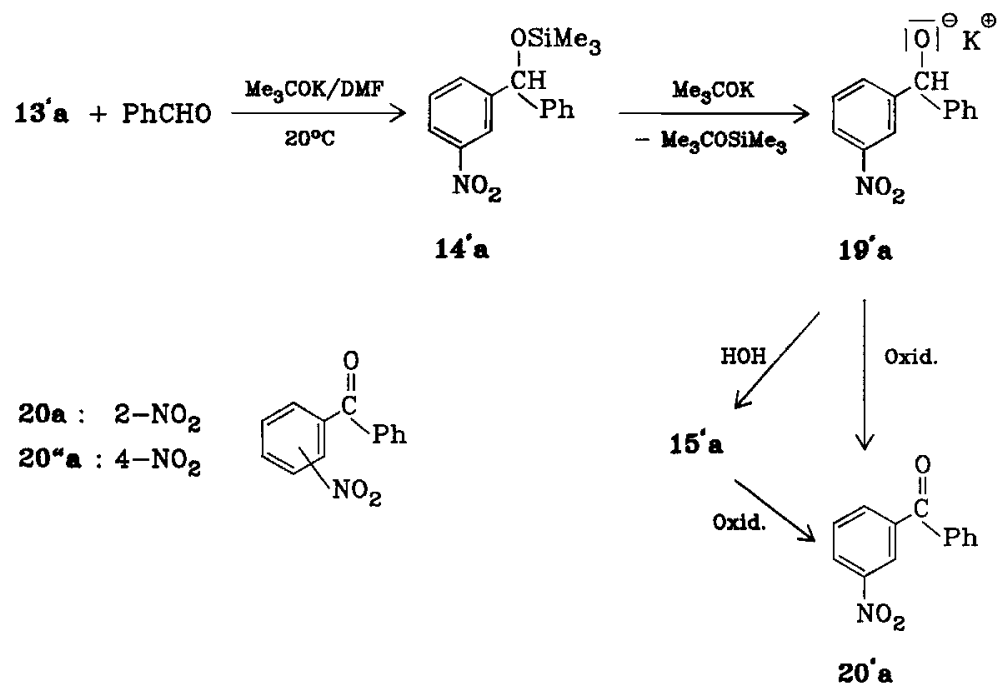

Die oxidative Konkurrenzreaktion zum Benzophenon-Derivat wird bei der Carbodesilylierung des 2-Isomeren 13a offensichtlich infolge der größeren Bildungsgeschwindigkeit des Trimethylsilylethers 14 a verhindert; getrennte Umsetzungen von 14 a mit 33 mol- $\% \mathrm{Me}_{3} \mathrm{COK}$ zeigten jedoch, daß auch 14 a zum entsprechenden Benzophenon 20 a oxidiert werden kann, allerdings unter drastischeren Reaktionsbedingungen: Nach $8 \mathrm{~h}$ Umsetzung bei $100^{\circ} \mathrm{C}$ in DMF waren gaschromatographisch weder Ausgangsprodukt 14a noch (2-Nitrophenyl)phenylmethanol (15a) nachweisbar, sondern lediglich 27\% 1-Benzoyl-2-nitrobenzol (20a).

Erwartungsgemäß reagiert das unsubstituierte (Trimethylsilyl)benzol basekatalysiert viel langsamer als die Acceptor-substituierten Benzolderivate. Mit Cäsiumfluorid als Katalysator erfolgt selbst bei $100^{\circ} \mathrm{C}$ in HMPT mit Benzaldehyd keine Carbodesilylierung, mit $\mathrm{Me}_{3} \mathrm{COK}$ werden zwar kleine Mengen an Benzhydrol erhalten, im wesentlichen reagiert der Benzaldehyd jedoch im Sinne einer Tischtschenko-Reaktion zu Benzoesäure-benzylester.

Der enorme Einfluß der Aryl- bzw. Heteroaryl-Anionen-Stabilisierung auf die Geschwindigkeit der basekatalysierten Carbodesilylierung wird auch aus den Reaktionen der (Trimethylsilyl)thiophene mit Benzaldehyd deutlich. Die Carbodesilylierung von 2-(Trimethylsilyl)thiophen (21) mit Benzaldehyd verlief mit 2 mol-\% $\mathrm{Me}_{3} \mathrm{COK}$ in DMF stark exotherm unter Rotfärbung des Reaktionsgemisches und Erwärmung auf $70^{\circ} \mathrm{C}$, nach $1 \mathrm{~h}$ wurden $80 \%$ 2-[Phenyl(trimethyl- 
siloxy)methyl]thiophen (22) isoliert. 3-(Trimethylsilyl)thiophen (21') zeigte erwartungsgemäß eine geringere Reaktivität gegenüber Benzaldehyd (basische H/DAustauschwerte von $21=3.5 \cdot 10^{-4}$ und von $21^{\prime}=1 \cdot 10^{-6}$ in $\mathrm{CH}_{3} \mathrm{OD} /$ $\left.\mathrm{CH}_{3} \mathrm{ONa} / 31^{\circ} \mathrm{C}\right)^{28)}$ und ergab in Gegenwart von $\mathrm{CsF}$ in HMPT nach $24 \mathrm{~h}$ bei $100^{\circ} \mathrm{C} \mathrm{zu} \mathrm{42 \%} \mathrm{3-[Phenyl(trimethylsiloxy)methyl]} \mathrm{thiophen} \mathrm{(22').}$

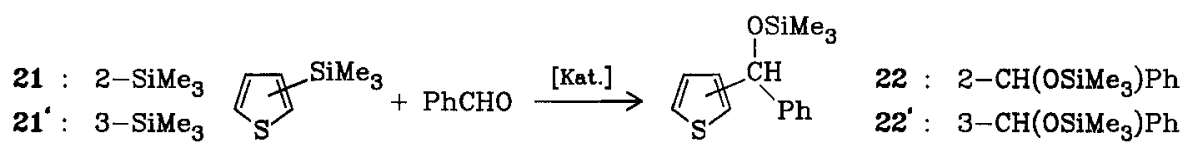

\section{Zur Reaktivität der basisch katalysierten Carbodesilylierung substituierter Phenyltrimethylsilane}

Bei Protodesilylierungsreaktionen meta- bzw. para-substituierter Phenyltrimethylsilane im basischen Medium wurde durch kinetische Messungen eine gute Korrelation zwischen den $\log k_{\text {rel }}$-Werten und den Hammettschen $\sigma_{m}$ - bzw. $\sigma_{p}$ Werten gefunden, wobei der hohe $\mathrm{Q}-$ Wert von $6.0^{8 \mathrm{~d})}$ auf das Vorliegen eines Übergangszustandes oder eines Zwischenproduktes mit hoher negativer Ladung hinweist.

Neuere Untersuchungen dieser Reaktionen, unter Einbeziehung ortho-substituierter Phenyltrimethylsilane, ergeben Reaktionsgeschwindigkeiten, die befriedigend mit den für die entsprechenden Aryl-Anionen berechneten Stabilitäten korrelieren $^{8 \mathrm{c})}$. Zur Ermittlung der Reaktivität substituierter Phenyltrimethylsilane bei der Umsetzung mit Benzaldehyd in Gegenwart anionischer Katalysatoren haben wir keine kinetischen Messungen durchgeführt, sondern lediglich kompetitive Umsetzungen ${ }^{29)}$ mit einigen der von uns eingesetzten Aryltrimethylsilane (Tab. 11, exp. Teil). Daraus ergibt sich folgende Reaktivitätsabstufung in Abhängigkeit von der Art und der Position des Substituenten am Phenyltrimethylsilan:

$$
2-\mathrm{NO}_{2} \gg 2-\mathrm{Cl} \sim 2-\mathrm{F}>3-\mathrm{NO}_{2} \geq 4-\mathrm{NO}_{2}
$$

Vergleicht man diese Befunde mit den Geschwindigkeiten der basisch katalysierten Protodesilylierung ${ }^{8 c)}$ bzw. den aus den Dissoziationskonstanten entsprechend substituierter Benzoesäuren ermittelten $\sigma-W^{-W_{t e n}{ }^{30} \text { ) }}$ (Tab. 5), so korreliert die Reaktivität der basisch katalysierten Carbodesilylierung sehr viel besser mit den $\sigma$-Werten als den Protodesilylierungsgeschwindigkeiten. Während die basische Protodesilylierung der 3- bzw. 4-Nitro-1-(trimethylsilyl)benzole deutlich rascher verläuft als die der 2-Chlor- bzw. 2-Fluor-Derivate, erfolgt die Carbodesilylierung in der genau umgekehrten Reihenfolge, d. h. in Übereinstimmung mit den entsprechenden $\sigma$-Werten.

Da für die basisch katalysierte Protodesilylierung eine gute Korrelation mit den berechneten Aryl-Anionen-Stabilitäten gefunden wurdd ${ }^{8 \mathrm{~b}, \mathrm{c}}$, könnte man aus diesen Ergebnissen den Schluß ziehen, daß bei der basisch katalysierten Carbodesilylierung der anionische Charakter der auftretenden Zwischenstufen weniger ausgeprägt ist, d. h. daß eine Unterstützung des Dissoziationsschrittes durch das Elektrophil gegeben ist.

Chem. Ber. 118 (1985) 
Tab. 5. Relative Reaktionsgeschwindigkeiten der basisch katalysierten Protodesilylierung von (Trimethylsilyl)benzolen ${ }^{8 c)}$ und $\sigma$-Werte substituierter Benzoesäuren ${ }^{30 \text { ) }}$

(Trimethyl- rel. Reaktionsgeschw.

silyl)benzol der Protodesilylierung

$\mathrm{Z}$

-benzoesäure $\quad \sigma-$ Werte

$\begin{array}{lrll}\mathrm{H} & 1 & \mathrm{H} & 0 \\ 2-\mathrm{NO}_{2} & 420000 & \text { 2-Nitro- } & 1.99 \\ \text { 3- } \mathrm{NO}_{2} & 9700 & \text { 3-Nitro- } & 0.71 \\ 4-\mathrm{NO}_{2} & 10300 & \text { 4-Nitro- } & 0.78 \\ 2-\mathrm{Cl} & 3960 & \text { 2-Chlor- } & 1.28 \\ 3-\mathrm{Cl} & 375 & \text { 3-Chlor- } & 0.37 \\ 4-\mathrm{Cl} & 38 & \text { 4-Chlor- } & 0.23 \\ 2-\mathrm{F} & 4000 & \text { 2-Fluor- } & 0.93 \\ \text { 2-MeO } & 6.3 & \text { 2-Methoxy- } & 0.12\end{array}$

Ein weiterer Befund, der diese Vorstellungen stützt und der zum Verständnis der Reaktionen von Arylsilanen mit Basen beiträgt, ist das Reaktionsverhalten des 2-Brom- (13f) bzw. 2-Iod-1-(trimethylsilyl)benzols. Mit der Bromverbindung wurden bei der basisch katalysierten Reaktion mit Benzaldehyd auch nach langen Reaktionszeiten nur minimale Ausbeuten an Carbodesilylierungsprodukt erhalten (Tab. 3), obwohl der $\sigma$-Wert für 2-Brombenzoesäure mit 1.35 angegeben ist ${ }^{30)}$ und demnach eine vergleichbare Reaktivität wie mit der Chlorverbindung zu erwarten gewesen wäre. Die Iodverbindung ergibt überhaupt kein Carbodesilylierungsprodukt mehr.

Dieses abweichende Verhalten ist eine Folge der besseren Austrittstendenz von Bromid bzw. Iodid gegenüber Fluorid bzw. Chlorid. Die Eliminierung zum Arin ist in diesen Fällen gegenüber der Reaktion mit Elektrophilen bevorzugt. Bei Umsetzungen von ortho-Halogen(trimethylsilyl)benzolen mit Basen unter Ausschluß von Elektrophilen wurde die Bildung von Arinen schon früher beschrieben ${ }^{31)}$; wir werden in einer späteren Arbeit genauer auf diese Konkurrenz zwischen Reaktionen mit Elektrophilen und Arinbildung eingehen ${ }^{32)}$.

Wir danken Herrn $R$. König, Institut für Siedlungswasserbau der Universität Stuttgart, für die Durchführung der Löslichkeitsmessungen mittels Atomabsorptionsspektrometrie sowie den Herren cand. chem. H. Kottmann und $J$. König für engagierte Mitarbeit im Rahmen des Forschungspraktikums. Unser Dank gilt ebenfalls der Deutschen Forschungsgemeinschaft sowie dem Fonds der Chemischen Industrie für die Förderung dieser Untersuchungen.

\section{Experimenteller Teil}

Gaschromatographie: Gerät 5700 A der Fa. Hewlett-Packard mit Flammenionisationsdetektor (FID), Integrator: Spectraphysics Minigrator. Trägergas $30 \mathrm{ml}$ Reinstickstoff/min, Glaskapillare $2.30 \mathrm{~m} \times 2 \mathrm{~mm}$. Stationäre Phase OV 101/5\% auf Gaschrom Q und OV 25/ $5 \%$ auf Gaschrom $Q$. Temperaturprogramm $100-300^{\circ} \mathrm{C}, 16^{\circ} \mathrm{C} / \mathrm{min}$. Die Identifizierung 
der Produkte erfolgte durch Koinjektion von Vergleichssubstanzen, die Ausbeuten wurden nach Einwaage einer definierten Menge an internem Standard (ca. $0.1 \mathrm{~g}$ Tetralin/mmol Reaktand) und mit Hilfe von Eichmessungen erhalten ${ }^{33}$. - Präparative Säulenchromatographie: nach Lit. ${ }^{34)}$ mit Glassäulen Typ C, $40 \times 4 \mathrm{~cm}$, gefüllt mit Kieselgel (Fa. Merck, 0.015-0.025 mm), ca. 6600 theor. Böden, UV-Detektor: Spektrochrom (Fa. Gilson). - ${ }^{1} \mathrm{H}$ NMR-Spektren: Geräte T 60, EM 360 der Firma Varian sowie HX 90 der Fa. Bruker. GC/MS-Spektren: Gaschromatograph: Carlo-Erba Fractovap 2151 AC-Special der Firma Brechbühler AG, Urdorf. - Massenspektrometer: Finnigan 4023 mit Incos-Datensystem 2300, Trennkapillare: $22 \mathrm{~m}$, Phase SE-25, $110 \mathrm{eV}$, Reaktandgasdruck 0.3 Torr (Methan oder Butan).

Kalium-tert-butylat ${ }^{35)}\left(\mathrm{Me}_{3} \mathrm{COK}\right)$ wurde frisch sublimiert, die anderen Katalysatoren wurden nach Trocknen über Phosphorpentoxid (bis zu $300^{\circ} \mathrm{C}$ ) mittels eines Vibrorührers im Ultraschallbad fein suspendiert eingesetzt. Tetraethylammoniumfluorid $\left(\mathrm{Et}_{4} \mathrm{NF} \cdot 2 \mathrm{H}_{2} \mathrm{O}\right)$ wurde aus Tetraethylammonium-tetrafluoroborat nach Lit. ${ }^{36)}$ synthetisiert. Der nach Entfernen des Lösungsmittels i. Vak. erhaltene farblose Feststoff wurde entweder mehrere Tage bei $70^{\circ} \mathrm{C}$ über $\mathrm{P}_{2} \mathrm{O}_{5}$ getrocknet oder durch Lösen in wasserfreiem Benzol/Acetonitril $(1: 1)$ bei $60^{\circ} \mathrm{C}$ und anschließendem Abdestillieren des Lösungsmittelgemisches i. Wasserstrahlvak. bei $60^{\circ} \mathrm{C}$ gereinigt. Nach fünfmaligem Wiederholen zeigte die zurückbleibende Substanz den Schmp. $80^{\circ} \mathrm{C}$ (Zers.) (Lit. ${ }^{37)}$ kein Schmp., keine Analysendaten).

$$
\begin{aligned}
& \left(\mathrm{C}_{2} \mathrm{H}_{5}\right)_{4} \mathrm{NF} \cdot 2 \mathrm{H}_{2} \mathrm{O}(185.3) \quad \text { Ber. C } 51.84 \mathrm{H} 13.05 \mathrm{~N} 7.59 \\
& \text { Gef. C } 50.36 \text { H } 13.05 \quad \text { N } 7.69
\end{aligned}
$$

Allgemeine Arbeitsweise: Sämtliche Reaktionen unter nucleophiler Katalyse werden unter Luft- und Feuchtigkeitsausschluß durchgeführt, Reaktionen, bei denen der Katalysator vorgelegt wird, in einem Einhalskolben, solche, bei denen der Katalysator zu den Edukten gegeben wird, in einem Zweihalskolben mit Feststoffdosierstück. Die Gefäße werden mit Magnetrührstäbchen und Katalysator fünfmal i. Vak. ausgeheizt und mit Stickstoff begast. Anschließend wird das Gasableitungsrohr mit Hahn unter Schutzgas (Stickstoff oder Argon) gegen ein Septum (rubber septum der Fa. Aldrich) ausgetauscht. Die Zugabe der Reaktanden und der Lösungsmittel sowie die Probeentnahmen erfolgen durch das Septum mit einer Spritze. Die Gemische werden die angegebene Zeit bei der angegebenen Temperatur gerührt und anschließend aufgearbeitet.

\section{Aufarbeitung}

Methode A: Der Katalysator wird über eine Vakuumfritte abgesaugt, aus der klaren Lösung das Lösungsmittel i. Wasserstrahlvak. über eine Kolonne abdestilliert und der Rückstand i. Vak. destilliert oder umkristallisiert.

Methode B: Der nach Methode A erhaltene Rückstand wird im genannten Eluens aufgenommen und über eine Glassäule ( $30 \mathrm{~cm}$ lang, gefüllt mit $60 \mu-K$ ieselgel der Fa. Merck) filtriert.

Methode C: Das Reaktionsgemisch wird mit Wasser oder verd. Säure 2 h gerührt und mit Ether extrahiert. Der Etherextrakt wird über Magnesiumsulfat getrocknet und im Rotationsverdampfer eingeengt.

Methode GC/A: Die dem Reaktionsgemisch entnommene Probe wird mit der 20-30fachen Menge Ether verdünnt. Nach Stehenlassen unter Verschluß bis zum Absetzen des Niederschlags wird mittels einer Pipette die klare Lösung abgesaugt und gaschromatographisch untersucht.

Chem. Ber. 118 (1985) 
Methode GC/B: Die dem Reaktionsgemisch entnommene Probe wird mit der dreifachen Menge 2proz. Salzsäure oder 5proz. Schwefelsäure hydrolysiert und mit Ether extrahiert. Aus der über Magnesiumsulfat getrockneten Etherphase werden Proben mit einer Pipette abgesaugt und gaschromatographisch untersucht.

Carbodesilylierung von 2-(Trimethylsilyl) benzothiazol (1) ${ }^{14 a)}$ und 1-Methyl-2-(trimethylsilyl)benzimidazol (2) ${ }^{14 \mathrm{~b})}$ mit Benzaldehyd in Gegenwart von $\mathrm{Me}_{3} \mathrm{COK}$

1) $\mathrm{Mit} 1 \mathrm{~mol}-\% \mathrm{Me}_{3} \mathrm{COK}$

a) $\mathrm{Zu} 0.0015 \mathrm{~g}(0.013 \mathrm{mmol}) \mathrm{Me}_{3} \mathrm{COK}$ in $1.5 \mathrm{ml} \mathrm{DMF}$ werden bei $-60^{\circ} \mathrm{C}$ nacheinander $0.269 \mathrm{~g}(1.3 \mathrm{mmol}) 1$ in $0.5 \mathrm{ml} \mathrm{DMF}$ und $0.138 \mathrm{~g}(1.3 \mathrm{mmol})$ Benzaldehyd in $0.5 \mathrm{ml} \mathrm{DMF}$ getropft, wobei sich das Gemisch schwach blau färbt. Nach 15 min Rühren bei $-60^{\circ} \mathrm{C}$ wird nach Methode GC/A aufgearbeitet. Ausb. 91\% 2-/Phenyl(trimethylsiloxy)methyl]benzothiazol (3).

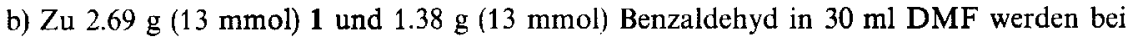
$-60^{\circ} \mathrm{C} 0.015 \mathrm{~g}(0.13 \mathrm{mmol}) \mathrm{Me}_{3} \mathrm{COK}$ gegeben. Nach $15 \mathrm{~min}$ Rühren bei $-60^{\circ} \mathrm{C}$ zeigt die gaschromatographische Untersuchung einer Probe vollständigen Umsatz an, das Gemisch wird auf Raumtemp. erwärmt, nach Methode A aufgearbeitet und der erstarrte Feststoff aus Heptan umkristallisiert. Ausb. 2.1 g (51\%) 3, Sdp. $124^{\circ} \mathrm{C} / 10^{-3}$ Torr, Schmp. $95-97^{\circ} \mathrm{C}$ (Heptan) (Lit. ${ }^{14 a)} 97-99^{\circ} \mathrm{C}$ ).

c) $\mathrm{Zu}$ der Suspension von $0.011 \mathrm{~g}(0.10 \mathrm{mmol}) \mathrm{Me}_{3} \mathrm{COK}$ in $20 \mathrm{ml} \mathrm{DMF}$ werden bei $-60^{\circ} \mathrm{C}$ nacheinander $2.04 \mathrm{~g}(10 \mathrm{mmol}) 2$ in $5 \mathrm{ml}$ DMF und $1.06 \mathrm{~g}(10 \mathrm{mmol})$ Benzaldehyd in $5 \mathrm{ml}$ DMF getropft, wobei sich das Gemisch schwach gelb färbt. Nach 15 min Rühren bei $-60^{\circ} \mathrm{C}$ wird eine Probe nach Methode GC/A aufgearbeitet, Ausb. 98\% 1-Methyl-2-/phenyl/trimethylsiloxy) methyl Jbenzimidazol (4). Das Reaktionsgemisch wird auf Raumtemp. erwärmt und nach Methode A aufgearbeitet. Ausb. $2.1 \mathrm{~g} \mathrm{(68 \% )} \mathrm{4,} \mathrm{Sdp.} 132-134^{\circ} \mathrm{C} / 0.08$ Torr (Lit. ${ }^{14 b)}$ $130^{\circ} \mathrm{C} / 0.05$ Torr).

2) Mit verschiedenen Mengen Me,COK: Jeweils $0.269 \mathrm{~g}(1.3 \mathrm{mmol}) 1$ und $0.138 \mathrm{~g}$ (1.3 mmol) Benzaldehyd werden in $4 \mathrm{ml} \mathrm{DMF} \mathrm{mit} 0.029 \mathrm{~g}(0.26 \mathrm{mmol}), 0.073 \mathrm{~g}(0.65 \mathrm{mmol})$ bzw. $0.146 \mathrm{~g}(1.3 \mathrm{mmol}) \mathrm{Me}_{3} \mathrm{COK} 15 \mathrm{~min}$ bei $-60^{\circ} \mathrm{C}$ gerührt. Es wird nach Methode $\mathrm{GC} / \mathrm{A}$ aufgearbeitet.

Tab. 6. Abnahme der Ausbeute an 2-[Phenyl(trimethylsiloxy)methyl]benzothiazol (3) in Abhängigkeit von der Konzentration an $\mathrm{Me}_{3} \mathrm{COK}$ bei der Carbodesilylierung von 2-(Trimethylsilyl)benzothiazol (1) mit Benzaldehyd

\begin{tabular}{lrrrr}
\hline mol-\% Me $\mathrm{COK}$ & 1 & 22 & 54 & 100 \\
Ausb. \% $\%^{\text {a) }} 3$ & 91 & 63 & 18 & $4^{\text {b) }}$ \\
\hline
\end{tabular}

a) Gaschromatographisch bestimmt. - b) Außerdem wurden 44\% 2-Benzothiazolylphenylmethanol (5), 52\% Benzothiazol (6) und (isotherm bei $70^{\circ} \mathrm{C}$ ) tert-Butyl(trimethylsily1)ether (8) sowie Bis(trimethylsilyl)ether (7) durch Koinjektion identifiziert.

3) Unter portionsweiser Zugabe von insgesamt $100 \mathrm{~mol} \% \mathrm{Me} \mathrm{C}_{3} \mathrm{COK}: \mathrm{Zu} 0.207 \mathrm{~g}(1.0 \mathrm{mmol})$ 1 und $0.106 \mathrm{~g}(1.0 \mathrm{mmol})$ Benzaldehyd in $3 \mathrm{ml} \mathrm{DMF}$ werden in drei Portionen bei $-60^{\circ} \mathrm{C}$ $0.112 \mathrm{~g}(1.0 \mathrm{mmol}) \mathrm{Me}_{3} \mathrm{COK}$ gegeben. Nach jeder Zugabe wird $5 \mathrm{~min}$ bei $-60^{\circ} \mathrm{C}$ gerührt und anschließend eine Probe nach Methode GC/A aufgearbeitet (Tab. 7).

Umsetzung von 2-[Phenyl(trimethylsiloxy)methyl]benzothiazol (3) mit der äquimolaren Menge $\mathrm{Me}_{3} \mathrm{COK}$ : $0.313 \mathrm{~g}$ (1.0 mmol) 3 und $0.112 \mathrm{~g}(1.0 \mathrm{mmol}) \mathrm{Me}_{3} \mathrm{COK}$ werden in $4 \mathrm{ml}$ 
Tab. 7. Prozentuale Produktverteilung bei der Carbodesilylierung von 2-(Trimethylsilyl)benzothiazol (1) mit Benzaldehyd unter portionsweiser Zugabe von insgesamt $100 \mathrm{~mol}-\%$ $\mathrm{Me}_{3} \mathrm{COK}$ in $\mathrm{DMF}$ bei $-60^{\circ} \mathrm{C}$

\begin{tabular}{cccc}
\hline $\mathrm{Me}_{3} \mathrm{COK}-$ Zugabe & 1. & 2. & 3. \\
\hline Produkte nach & $5 \mathrm{~min}$ & $10 \mathrm{~min}$ & $15 \mathrm{~min}$ \\
\hline $\begin{array}{l}\text { 2-[Phenyl(trimethyl- } \\
\text { siloxy)methyl]- } \\
\text { benzothiazol (3) } \\
\begin{array}{l}\text { 2-Benzothiazolyl- } \\
\text { phenylmethanol (5) }\end{array}\end{array}$ & 84 & 32 & 4 \\
\hline
\end{tabular}

DMF $1 \mathrm{~h}$ bei $-60^{\circ} \mathrm{C}$ gerührt; Aufarbeitung nach Methode GC/A. Ausb. 35\% 2-Benzothiazolylphenylmethanol (5), daneben wurden noch $11 \% 3$ nachgewiesen.

Umsetzung von 1 mit der äquimolaren Menge $\mathrm{Me}_{3} \mathrm{COK}: 0.269 \mathrm{~g}(1.3 \mathrm{mmol}) 1$ und $0.146 \mathrm{~g}$ (1.3 mmol) $\mathrm{Me}_{3} \mathrm{COK}$ in $4 \mathrm{ml} \mathrm{DMF}$ werden $15 \mathrm{~min}$ bei $-60^{\circ} \mathrm{C}$ gerührt. Nach Methode $\mathrm{GC} /$ A wird aufgearbeitet. Ausb. ca. $90 \%$ tert-Butyl (trimethylsilyl)ether (8) und ca. $20 \%$ Benzothiazol (6). 1 konnte nicht mehr nachgewiesen werden.

Carbodesilylierung von 1 mit Benzaldehyd in Gegenwart verschiedener Katalysatoren (jeweils $10 \mathrm{~mol}-\%$ ): Die Suspension des getrockneten (s. vorstehend) und fein verriebenen Katalysators (jeweils $0.10 \mathrm{mmol}=11.2 \mathrm{mg} \mathrm{Me} 3 \mathrm{COK}, 13 \mathrm{mg}$ PhOK, $9.8 \mathrm{mg} \mathrm{MeCO} \mathrm{K}_{2}$, $8.4 \mathrm{mg} \mathrm{HCO} 2 \mathrm{~K}, 5.8 \mathrm{mg} \mathrm{KF}, 7.4 \mathrm{mg} \mathrm{KCl}, 44.2 \mathrm{mg}$ Aliquat $336^{\mathbf{R}}, 18.5 \mathrm{mg} \mathrm{Et}{ }_{4} \mathrm{NF} \cdot 2 \mathrm{H}_{2} \mathrm{O}$, $19.6 \mathrm{mg} \mathrm{Et}_{4} \mathrm{NBr}(0.093 \mathrm{mmol}), 2.6 \mathrm{mg} \mathrm{LiF}, 15.2 \mathrm{mg} \mathrm{CsF}, 6.6 \mathrm{mg} \mathrm{MeCO}_{2} \mathrm{Li}, 30.1 \mathrm{mg}$ $\mathrm{MeCO}_{2} \mathrm{NBu}_{4}$ und $\left.31.8 \mathrm{mg}\left(\mathrm{MeCO}_{2}\right)_{2} \mathrm{Hg}\right)$ in $3 \mathrm{ml} \mathrm{DMF}$ wird bei Raumtemp. $1 \mathrm{~h}$ gerührt. Anschließend werden bei der angegebenen Temp. die Lösungen von $0.207 \mathrm{~g}(1.00 \mathrm{mmol}) \mathrm{I}$ in $0.5 \mathrm{ml} \mathrm{DMF}$ und $0.106 \mathrm{~g}(1.00 \mathrm{mmol})$ Benzaldehyd in $0.5 \mathrm{ml}$ DMF unter Rühren zugegeben. Nach der angegebenen Zeit werden Proben entnommen und nach Methode GC/A aufgearbeitet. Ergebnisse s. Tab. 1.

Vergleichende Carbodesilylierung von 1 und 2 mit Benzaldehyd in DMF in Gegenwart verschiedener Katalysatoren: Jeweils $0.207 \mathrm{~g}(1.00 \mathrm{mmol}) 1 \mathrm{bzw} .0 .204 \mathrm{~g}(1.00 \mathrm{mmol}) 2$ werden mit $0.106 \mathrm{~g}$ (1.00 mmol) Benzaldehyd und $0.0132 \mathrm{~g}$ (0.100 mmol) PhOK bzw. $0.098 \mathrm{~g}(1.00$ mmol) $\mathrm{MeCO}_{2} \mathrm{~K}$ in $2 \mathrm{ml}$ DMF umgesetzt. Nach der angegebenen Zeit wird nach Methode GC/A aufgearbeitet. Ergebnisse s. Tab. 2.

Löslichkeitsbestimmung verschiedener Katalysatorsalze in DMF: Die Untersuchungen erfolgten mit einem Perkin-Elmer 306 Atomabsorptionsspektrometer. Kalium: $383 \mathrm{~nm}$ (vis) Spalt $4(14 \AA)=766.5 \mathrm{~nm}$; Lithium: $335 \mathrm{~nm}$ (vis) Spalt $4(14 \AA)=670.8 \mathrm{~nm}$. Verbrennung: Luft-Acetylen-Gemisch 30:18. Standard: Kalium- und Lithium-Salze von Cyclohexanbuttersäure (Merck). Konzentrationsreihen von 1.0, 2.5, 5.0, 7.5, $10.0 \mathrm{mg}$ Metall-Ionen pro Liter DMF (Tab. 8).

Umsetzung von 1 mit $\mathrm{Me}_{3} \mathrm{COK}$ in Gegenwart von Sauerstoff: In die Suspension von $0.280 \mathrm{~g}$ (2.5 mmol) $\mathrm{Me}_{3} \mathrm{COK}$ in $10 \mathrm{ml} \mathrm{DMF}$ leitet man bei $-60^{\circ} \mathrm{C}$ unter Rühren $15 \mathrm{~min}$ Sauerstoff, tropft dann unter weiterem Einleiten von Sauerstoff langsam die Lösung von $1.04 \mathrm{~g}$ (5.0 mmol) 1 in $7 \mathrm{ml} \mathrm{DMF} \mathrm{zu,} \mathrm{entfernt} \mathrm{das} \mathrm{Kühlbad} \mathrm{und} \mathrm{rührt} \mathrm{noch} 4 \mathrm{~h}$ unter weiterem Einleiten von Sauerstoff bei Raumtemp. Der farblose Niederschlag wird abgesaugt, mit Wasser und wenig Ether gewaschen und getrocknet. Ausb. $0.33 \mathrm{~g}(49 \%) 2,2^{\prime}$-Bibenzothiazol (9), Schmp. $310-312^{\circ} \mathrm{C}$ ( Lit. $^{38)} 304^{\circ} \mathrm{C}$ ).

Chem. Ber. 118 (1985) 
Tab. 8. Löslichkeiten verschiedener Katalysatorsalze in Dimethylformamid bei $25^{\circ} \mathrm{C}$

\begin{tabular}{lclr}
\hline Salz & $\begin{array}{c}\text { mmol/ } \\
\text { 100 ml DMF }\end{array}$ & \multicolumn{1}{c}{ Salz } & $\begin{array}{r}\text { mmol/ } \\
\text { 100 ml DMF }\end{array}$ \\
\hline $\mathrm{Me}_{3} \mathrm{COK}$ & 6.1 & $\mathrm{KF}$ & $\mathbf{0 . 1 4}$ \\
$\mathrm{PhOK}^{\mathrm{KeCl}}$ & 2.2 & $\mathrm{LiF}$ & 0.32 \\
$\mathrm{HCO}_{2} \mathrm{~K}$ & 2.8 & $\mathrm{Et}_{4} \mathrm{NBr}$ & 0.06 \\
$\mathrm{MeCO}_{2} \mathrm{Li}$ & 4.1 & $\mathrm{Bu}_{4} \mathrm{NCl}$ & 240.00 \\
\hline
\end{tabular}

Basekatalysierter Austausch der Trialkylsilyl-Substituenten von 2-(Triethylsilyl)benzothiazol $(10)^{39)}$ und 2,3,4-Trichlor-5-(trimethylsilyl)thiophen $(11)^{40)}$ : Lösungen von jeweils $0.249 \mathrm{~g}$ $(1.00 \mathrm{mmol}) 10$ und $0.259 \mathrm{~g}(1.00 \mathrm{mmol}) 11$ in $2 \mathrm{ml} \mathrm{DMF}$ werden mit $0.011 \mathrm{~g}(0.10 \mathrm{mmol})$ $\mathrm{Me}_{3} \mathrm{COK}$ versetzt und $2 \mathrm{~h}$ bei $-60^{\circ} \mathrm{C}$ gerührt bzw. mit $0.012 \mathrm{~g}(0.20 \mathrm{mmol})$ Kaliumfluorid versetzt und $24 \mathrm{~h}$ bei $20^{\circ} \mathrm{C}$ gerührt. Nach Methode GC/A wird aufgearbeitet. Es werden jeweils Gemische aus 10, 11, 1 und 2,3,4-Trichlor-5-(triethylsilyl)thiophen (12) durch GC/MS nachgewiesen.

Tab. 9. GC/MS-spektroskopische Daten eines Gemisches aus 2-(Trimethylsilyl)benzothiazol (1), 2-(Triethylsilyl)benzothiazol (10), 2,3,4-Trichlor-5-(trimethylsilyl)thiophen (11) und 2,3,4-Trichlor-5-(triethylsilyl)thiophen (12)

\begin{tabular}{clll}
\hline Verbindung & $\begin{array}{c}(\mathbf{M H})^{+} \\
\text {(Intensität) }^{*}\end{array}$ & $\begin{array}{c}\text { (MH) } \\
\text { (Intensität) }\end{array}$ & R \\
\hline 1 & $208(100)$ & $192(70)$ & $\mathrm{CH}_{3}$ \\
10 & $250(100)$ & $220(83)$ & $\mathrm{C}_{2} \mathrm{H}_{5}$ \\
11 & $259(11)$ & $243(58)$ & $\mathrm{CH}_{3}$ \\
& $261(10)$ & $245(62)$ & \\
& $263(1)$ & $247(17)$ & $\mathrm{C}_{2} \mathrm{H}_{5}$ \\
12 & $265(1)$ & $249(<1)$ & \\
& 301 & $271(13)$ & \\
& 303 & $273(13)$ & $(2)$ \\
\end{tabular}

\section{Carbodesilylierung 2-substituierter (Trimethylsilyl)benzole 13}

1. Von 2-Nitro-1-(trimethylsilyl) benzol (13a) ${ }^{41)}$

a) Man läßt zu der Suspension von $0.011 \mathrm{~g}(0.10 \mathrm{mmol}) \mathrm{Me}_{3} \mathrm{COK}$ in $3 \mathrm{ml} \mathrm{DMF}$ bei $-60^{\circ} \mathrm{C} 0.195 \mathrm{~g}(1.0 \mathrm{mmol}) 13 \mathrm{a}$ und $0.106 \mathrm{~g}(1.0 \mathrm{mmol})$ Benzaldehyd tropfen - hierbei färbt sich das Gemisch grün -, rührt $1 \mathrm{~h}$ bei $-60^{\circ} \mathrm{C}$ und arbeitet nach $\mathrm{GC} / \mathrm{A}$ auf. Ausb. $92 \%$ 2-Nitro-1-[phenyl(trimethylsiloxy) methyl]benzol (14a).

b) Man gibt zu $3.91 \mathrm{~g} \mathrm{(20} \mathrm{mmol)} 13 \mathrm{a}$ und $2.12 \mathrm{~g} \mathrm{(20} \mathrm{mmol)} \mathrm{Benzaldehyd} \mathrm{in} 25 \mathrm{ml} \mathrm{DMF}$ unter Rühren bei Raumtemp. portionsweise $0.083 \mathrm{~g}(0.74 \mathrm{mmol}) \mathrm{Me}_{3} \mathrm{COK}$ - hierbei färbt sich das Gemisch unter Erwärmen tiefrot -, rührt $1 \mathrm{~h}$ bei Raumtemp. (nach $\mathrm{GC}$ einer Probe quantitativer Umsatz), arbeitet nach Methode A auf und destilliert den öligen Rück- 
stand. Ausb. $4.81 \mathrm{~g}(80 \%)$ 14a, Sdp. $136-138^{\circ} \mathrm{C} / 0.1$ Torr. $-{ }^{1} \mathrm{H}-\mathrm{NMR}\left(\mathrm{CDCl}_{3}\right): \delta=0.07$ (d, $\left.9 \mathrm{H}, \mathrm{Si}\left(\mathrm{CH}_{3}\right)_{3}\right), 6.57$ (s, CH), $7.20-8.00(\mathrm{~m}, 9$ aromat. $\mathrm{H})$.

\section{$\mathrm{C}_{16} \mathrm{H}_{19} \mathrm{NO}_{3} \mathrm{Si}$ (301.4) Ber. C 63.75 H 6.35 N 4.64 Gef. C 64.02 H 6.38 N 4.94}

Zum Strukturbeweis von $14 \mathrm{a}$ wird die Lösung von $0.54 \mathrm{~g}(5.0 \mathrm{mmol})$ Chlortrimethylsilan in $2 \mathrm{ml}$ Ether unter Kühlen und Rühren zu einer Lösung von $1.15 \mathrm{~g}(5.0 \mathrm{mmol})$ (2-Nitrophenyl)phenylmethanol (15a $)^{42)}, 0.40 \mathrm{~g}(5.0 \mathrm{mmol})$ Pyridin und $5 \mathrm{ml}$ Ether getropft. Nach Rühren über Nacht wird das Gemisch abfiltriert, das Filtrat im Rotationsverdampfer eingeengt und der Rückstand i. Vak. destilliert. Ausb. $1.1 \mathrm{~g}(73 \%) 14 \mathrm{a}, \mathrm{Sdp} .129-131{ }^{\circ} \mathrm{C} / 5$. $10^{-3}$ Torr, ${ }^{1} \mathrm{H}$-NMR-spektroskopisch identisch mit der durch Carbodesilylierung von 13a erhaltenen Substanz. - $1.51 \mathrm{~g}$ (5.0 mmol) $14 \mathrm{a}$ werden mit $2.5 \mathrm{ml}$ Methanol, $0.5 \mathrm{ml}$ Wasser und 1 Tropfen konz. Salzsäure $4 \mathrm{~h}$ unter Rückfluß erhitzt. Das Lösungsmittel wird abdestilliert, der ölige Rückstand über eine Kieselgelsäule mit Petrolether/Ethylacetat (1:1) filtriert, das Filtrat mit Petrolether versetzt und der ausfallende farblose Feststoff aus Petrolether umkristallisiert. Ausb. $0.75 \mathrm{~g}(66 \%)$ 15a, Schmp. $63^{\circ} \mathrm{C}$ (Lit. ${ }^{42 \mathrm{a})} 59-60^{\circ} \mathrm{C}$ ).

2. Von 2-Chlor-1-(trimethylsilyl) benzol (13b), das nach Lit. ${ }^{43)}$ dargestellt wird: $\mathrm{Zu}$ der auf $75^{\circ} \mathrm{C}$ erwärmten Suspension von $35.73 \mathrm{~g}(1.47 \mathrm{~mol})$ Magnesium und $159.72 \mathrm{~g}(1.47 \mathrm{~mol})$ Chlortrimethylsilan in $450 \mathrm{ml} \mathrm{HMPT}$ gibt man unter Rühren $215.86 \mathrm{~g}$ (1.47 mol) 1,2-Dichlorbenzol, rührt $48 \mathrm{~h}$ bei $75-80^{\circ} \mathrm{C}$, rührt das abgekühlte Gemisch in 2.21 einer mit Eis versetzten wäBrigen Lösung von $9 \mathrm{~g}$ Natriumhydrogencarbonat und extrahiert mit Ether. Der Etherextrakt wird über Magnesiumsulfat getrocknet, im Rotationsverdampfer eingeengt, der Rückstand i. Wasserstrahlvak. destilliert und die im Siedebereich von $67-95^{\circ} \mathrm{C} / 12$ Torr

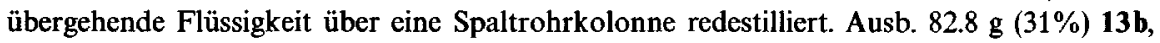
Sdp. $99^{\circ} \mathrm{C} / 21$ Torr (Lit. ${ }^{44)} 89^{\circ} \mathrm{C} / 15$ Torr).

a) $\mathrm{Zu}$ der Suspension von $0.044 \mathrm{~g}(0.40 \mathrm{mmol}) \mathrm{Me}_{3} \mathrm{COK}$ in $3 \mathrm{ml} \mathrm{DMF}$ läßt man bei $-30^{\circ} \mathrm{C}$ die Lösung von $0.370 \mathrm{~g}(2.0 \mathrm{mmol}) 13 \mathrm{~b}$ und $0.212 \mathrm{~g}(2.0 \mathrm{mmol})$ Benzaldehyd in $1 \mathrm{ml}$ DMF tropfen, rührt noch $1 \mathrm{~h}$ bei $-30^{\circ} \mathrm{C}$ und arbeitet nach Methode GC/A auf. Ausb. $80 \%$ 2-Chlor-1-[phenyl(trimethylsiloxy) methyl]benzol (14b).

b) Man gibt zu $3.70 \mathrm{~g}(20 \mathrm{mmol}) 13 \mathrm{~b}$ und $2.12 \mathrm{~g}(20 \mathrm{mmol})$ Benzaldehyd in $25 \mathrm{ml} \mathrm{DMF}$ portionsweise bei Raumtemp. $0.12 \mathrm{~g}$ (1.07 mmol) $\mathrm{Me}_{3} \mathrm{COK}$, rührt $90 \mathrm{~min}$ bei Raumtemp. (nach GC vollständiger Umsatz) und arbeitet nach Methode A auf. Ausb. $4.28 \mathrm{~g}(74 \%) \mathbf{1 4 b}$, Sdp. $93^{\circ} \mathrm{C} / 5 \cdot 10^{-3}$ Torr. $-{ }^{1} \mathrm{H}-\mathrm{NMR}\left(\mathrm{CDCl}_{3}\right): \delta=0.07$ (s, $\left.9 \mathrm{H}, \mathrm{Si}\left(\mathrm{CH}_{3}\right)_{3}\right), 6.22(\mathrm{~s}, \mathrm{CH})$, $7.0-7.7$ (m, 9 aromat. $\mathrm{H})$.

\section{$\mathrm{C}_{9} \mathrm{H}_{19} \mathrm{ClOSi}(290.9)$ Ber. C 66.07 H 6.58 Cl 12.18 Gef. C 66.30 H $6.60 \mathrm{Cl} 12.43$}

Zum Strukturbeweis von $14 \mathrm{~b}$ werden $2.19 \mathrm{~g}(10 \mathrm{mmol})$ (2-Chlorphenyl)phenylmethanol $(15 b)^{45)}$ mit $1.09 \mathrm{~g}(10 \mathrm{mmol})$ Chlortrimethylsilan in $0.79 \mathrm{~g}(10 \mathrm{mmol})$ Pyridin und $10 \mathrm{ml}$ Ether, wie bei der Darstellung von 14 a beschrieben, silyliert. Ausb. $1.83 \mathrm{~g}(63 \%) \mathbf{1 4 b}$, Sdp. $93^{\circ} \mathrm{C} / 5 \cdot 10^{-3}$ Torr, ${ }^{1} \mathrm{H}-\mathrm{NMR}$-spektroskopisch identisch mit der durch Carbodesilylierung von $13 \mathrm{~b}$ erhaltenen Substanz. - Die Hydrolyse von $1.45 \mathrm{~g}(5.0 \mathrm{mmol}) \mathbf{1 4 b} \mathrm{mit} 2.5 \mathrm{ml} \mathrm{Me}-$ thanol, $0.5 \mathrm{ml}$ Wasser und 1 Tropfen konz. Salzsäure analog der von 14 a zu 15a (s. vorstehend) ergibt $0.82 \mathrm{~g}(75 \%) 15 \mathrm{~b}$, Schmp. $66^{\circ} \mathrm{C}\left(\right.$ Lit. $\left.^{45)} 65-66^{\circ} \mathrm{C}\right)$.

3. Von 2-Fluor-1-(trimethylsilyl)benzol (13c), das nach Lit. ${ }^{43)}$ dargestellt wird: $\mathrm{Zu}$ dem auf $80^{\circ} \mathrm{C}$ erwärmten Gemisch aus $5.83 \mathrm{~g}(0.24 \mathrm{~mol})$ Magnesium und $26.08 \mathrm{~g}(0.24 \mathrm{~mol})$ Chlortrimethylsilan in $70 \mathrm{ml}$ HMPT läßt man unter Rühren die Lösung von $23.5 \mathrm{~g}(0.18 \mathrm{~mol})$ 1-Chlor-2-fluorbenzol in $70 \mathrm{ml}$ HMPT tropfen, rührt $96 \mathrm{~h}$ bei $100^{\circ} \mathrm{C}$ und arbeitet wie bei der Darstellung von $13 \mathrm{~b}$ beschrieben auf. Ausb. $9.26 \mathrm{~g} \mathrm{(31 \% )} \mathrm{13c,} \mathrm{Sdp.} 50-51^{\circ} \mathrm{C} / 13$ Torr

Chem. Ber. 118 (1985) 
(Lit. $\left.{ }^{44)} 167-168^{\circ} \mathrm{C}\right) .-{ }^{1} \mathrm{H}-\mathrm{NMR}\left(\mathrm{CDCl}_{3}\right): \delta=0.31$ (s, $\left.9 \mathrm{H}, \quad \mathrm{Si}\left(\mathrm{CH}_{3}\right)_{3}\right), 6.80-7.57$ (m, 4 aromat. $\mathrm{H}$ ).

$$
\mathrm{C}_{9} \mathrm{H}_{13} \mathrm{FSi} \text { (168.3) Ber. C } 64.23 \text { H } 7.78 \text { Gef. C } 64.32 \text { H } 8.01
$$

$\mathrm{Zu} 6.73 \mathrm{~g}$ (40 mmol) $13 \mathrm{c}$ und $4.24 \mathrm{~g}$ (40 mmol) Benzaldehyd in $40 \mathrm{ml} \mathrm{DMF}$ werden bei Raumtemp. auf einmal $0.224 \mathrm{~g}(2.0 \mathrm{mmol}) \mathrm{Me}_{3} \mathrm{COK}$ gegeben, wobei sich das Gemisch unter Erwärmen auf ca. $50^{\circ} \mathrm{C}$ rot färbt. Nach $1 \mathrm{~h}$ Rühren bei Raumtemp. wird nach Methode A aufgearbeitet und der Rückstand destilliert. Ausb. $8.3 \mathrm{~g}(76 \%)$ 2-Fluor-1-/phenyl (trimethylsiloxy)methyl Jbenzol (14c), Sdp. $84^{\circ} \mathrm{C} / 3 \cdot 10^{-3}$ Torr. $-{ }^{1} \mathrm{H}-\mathrm{NMR}\left(\mathrm{CDCl}_{3}\right): \delta=0.10(\mathrm{~s}, 9 \mathrm{H}$, $\left.\mathrm{Si}\left(\mathrm{CH}_{3}\right)_{3}\right), 6.17(\mathrm{~s}, \mathrm{CH}), 6.77-7.74(\mathrm{~m}, 9$ aromat. $\mathrm{H})$.

$$
\mathrm{C}_{16} \mathrm{H}_{19} \text { FOSi (274.4) Ber. C } 70.03 \text { H } 6.97 \text { Gef. C } 69.64 \text { H } 7.10
$$

4. Von 2-Phenoxy-1-(trimethylsilyl)benzol $(13 \mathrm{~d})^{46)}$ : $\mathrm{Zu} 3.63 \mathrm{~g}(15 \mathrm{mmol}) 13 \mathrm{~d}$ und $1.59 \mathrm{~g}$ (15 mmol) Benzaldehyd in $40 \mathrm{ml}$ DMF gibt man bei Raumtemp. unter Rühren $0.168 \mathrm{~g}$ (2.11 $\mathrm{mmol}) \mathrm{Me}{ }_{3} \mathrm{COK}$, rührt $2 \mathrm{~h}$ bei Raumtemp., arbeitet nach Methode A auf und destilliert den Rückstand. Ausb. $3.55 \mathrm{~g}(68 \%)$ 2-Phenoxy-1-[phenyl(trimethylsiloxy) methyljbenzol (14d), Sdp. $122-124^{\circ} \mathrm{C} / 2 \cdot 10^{-2}$ Torr. $-{ }^{1} \mathrm{H}-\mathrm{NMR}\left(\mathrm{CDCl}_{3}\right): \delta=0.04\left(\mathrm{~s}, 9 \mathrm{H}, \mathrm{Si}\left(\mathrm{CH}_{3}\right)_{3}\right), 6.21$ (s, CH), $6.70-7.80$ (m, 14 aromat. H).

\section{$\mathrm{C}_{22} \mathrm{H}_{24} \mathrm{O}_{2} \mathrm{Si}(348.5) \quad$ Ber. C 75.81 H 6.94 Gef. C 75.60 H 6.89}

\section{Von 2-(Phenylsulfonyl)-1-(trimethylsilyl)benzol (13e)}

13e: Zu dem aus $17.13 \mathrm{~g}(0.125 \mathrm{~mol})$ 1-Brombutan, $2.10 \mathrm{~g}(0.303 \mathrm{~mol})$ Lithium und $21.83 \mathrm{~g}$ $(0.10 \mathrm{~mol})$ Diphenylsulfon in $250 \mathrm{ml}$ Ether nach Lit. $^{47)}$ synthetisierten 2-Lithiumdiphenylsulfon läßt man bei $-40^{\circ} \mathrm{C}$ unter Rühren $10.86 \mathrm{~g}(0.10 \mathrm{~mol})$ Chlortrimethylsilan tropfen, läßt dann das Gemisch unter Rühren auf Raumtemp. erwärmen, gibt $500 \mathrm{ml}$ Chloroform zu, saugt vom ausgefallenen Lithiumchlorid ab, engt das Filtrat im Rotationsverdampfer ein und destilliert den Rückstand i. Vak. Ausb. $20.4 \mathrm{~g}(70 \%)$ 13e, Sdp. $132-134^{\circ} \mathrm{C} / 10^{-3}$ Torr. $-{ }^{1} \mathrm{H}-\mathrm{NMR}\left(\mathrm{CDCl}_{3}\right): \delta=0.45\left(\mathrm{~s}, 9 \mathrm{H}, \mathrm{Si}\left(\mathrm{CH}_{3}\right)_{3}\right), 7.38-8.10(\mathrm{~m}, 9$ aromat. $\mathrm{H})$.

\section{$\mathrm{C}_{15} \mathrm{H}_{18} \mathrm{O}_{2} \mathrm{SSi}(290.5) \quad$ Ber. C $62.02 \quad \mathrm{H} 6.25$ S 11.03 Gef. C 62.04 H $6.39 \mathrm{~S} 10.93$}

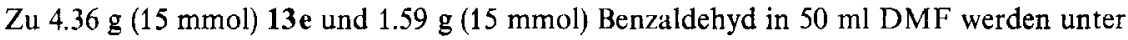
Rühren bei Raumtemp. auf einmal $0.118 \mathrm{~g}(1.05 \mathrm{mmol}) \mathrm{Me}_{3} \mathrm{COK}$ gegeben, wobei eine exotherme Reaktion unter Braunfärbung des Gemisches erfolgt. Nach 1 h Rühren wird nach Methode A aufgearbeitet, das erhaltene Destillat mit Petrolether/Essigester (20:1) versetzt und der farblose Feststoff abfiltriert. Ausb. $5.77 \mathrm{~g}$ (97\%) 2-(Phenylsulfonyl)-1-[phenyl (trimethylsiloxy)methyl Jbenzol (14e). Schmp. 85-90 ${ }^{\circ} \mathrm{C}$. $-{ }^{1} \mathrm{H}-\mathrm{NMR}\left(\mathrm{CDCl}_{3}\right): \delta=0.03(\mathrm{~s}, 9 \mathrm{H}$, $\left.\mathrm{Si}\left(\mathrm{CH}_{3}\right)_{3}\right), 6.86$ (s, $\left.\mathrm{CH}\right), 6.99-8.20$ (m, 14 aromat. $\mathrm{H}$ ).

$$
\mathrm{C}_{22} \mathrm{H}_{24} \mathrm{O}_{3} \mathrm{SSi} \text { (396.6) Ber. C } 66.63 \text { H } 6.10 \text { S } 8.80 \text { Gef. C } 66.46 \quad \mathrm{H} 6.03 \text { S } 7.80
$$

6. Von 2-Brom-1-(trimethylsilyl) benzol (13f), das modifiziert nach Lit. ${ }^{41)}$ dargestellt wurde: Man gibt zu der Lösung von $24.47 \mathrm{~g}(0.11 \mathrm{~mol})$ 1,2-Bis(trimethylsilyl)benzol in $150 \mathrm{ml}$ Chloroform unter Rühren bei $-78^{\circ} \mathrm{C}$ innerhalb $2.5 \mathrm{~h}$ die Lösung von $17.58 \mathrm{~g}(0.11 \mathrm{~mol})$ Brom in $150 \mathrm{ml}$ Chloroform, rührt noch $1 \mathrm{~h}$ bei $-78^{\circ} \mathrm{C}$, gibt nach Erwärmen auf Raumtemp. $100 \mathrm{ml}$ Ether $z u$, trennt die organische Phase $a b$, wäscht sie mit gesättigter wäßriger Natriumhydrogencarbonat-Lösung und trocknet sie über Magnesiumsulfat. Das Lösungsmittel wird im Rotationsverdampfer entfernt, der Rückstand destilliert und die bei $117-122^{\circ} \mathrm{C} /$ 22 Torr übergehende Flüssigkeit $(12.66 \mathrm{~g})$ redestilliert. Ausb. $9.18 \mathrm{~g}$ (36\%) 13f, Sdp. $90-92^{\circ} \mathrm{C} / 11$ Torr (Lit. ${ }^{41)} 77^{\circ} \mathrm{C} / 3.5$ Torr). Das Produkt enthält ca. $10 \%$ 1- und 3-BromIsomere $\left({ }^{1} \mathrm{H}-\mathrm{NMR}\right.$-spektroskopisch bestimmt). 
$4.81 \mathrm{~g}(21 \mathrm{mmol}) 13 \mathrm{f}$ werden mit $2.23 \mathrm{~g}(21 \mathrm{mmol})$ Benzaldehyd und $1.60 \mathrm{~g}(10.5 \mathrm{mmol})$ Cäsiumfluorid in $20 \mathrm{ml} \mathrm{HMPT} 24 \mathrm{~h}$ bei $100^{\circ} \mathrm{C}$ gerührt. Es wird nach Methode C aufgearbeitet. Zur vollständigen Hydrolyse wird das Rohprodukt mit $4 \mathrm{ml} 4$ proz. Salzsäure in $35 \mathrm{ml}$ Methanol $16 \mathrm{~h}$ bei $70^{\circ} \mathrm{C}$ gerührt, anschließend wird das Lösungsmittel im Rotationsverdampfer entfernt, der Rückstand in Ether aufgenommen und die etherische Lösung über Magnesiumsulfat getrocknet. Das Lösungsmittel wird im Rotationsverdampfer entfernt, der Rückstand zunächst über eine Kieselgelsäule mit Petrolether/Essigester (20:1) filtriert und anschließend mittels Mitteldrucksäulenchromatographie mit demselben Eluens gereinigt. Ausb. $0.66 \mathrm{~g}(12 \%)$ (2-Bromphenyl) phenylmethanol (15f), ${ }^{1} \mathrm{H}$-NMR-spektroskopisch identisch mit authentischer Substan $z^{48}$.

7. Von 2-Methoxy-1-(trimethylsilyl)benzol $(13 \mathrm{~g})^{43)}: 9.02 \mathrm{~g}(50 \mathrm{mmol}) \mathbf{1 3} \mathrm{g}$ und $5.30 \mathrm{~g}(50$ mmol) Benzaldehyd werden in $50 \mathrm{ml}$ HMPT mit $0.76 \mathrm{~g}$ (5.0 mmol) Cäsiumfluorid $134 \mathrm{~h}$ bei $100^{\circ} \mathrm{C}$ gerührt. Nach Methode $\mathrm{C}$ wird aufgearbeitet. Das erhaltene 2:1-Gemisch aus 2-Methoxy-1-(phenyl(trimethylsiloxy) methyl/benzol (14g) und (2-Methoxyphenyl)phenylmethanol (15g) wird in einem Gemisch aus $60 \mathrm{ml}$ Methanol und $9 \mathrm{ml}$ Wasser über Nacht bei Raumtemp. gerührt. Anschließend wird das Lösungsmittel abgezogen, der Rückstand in Ether aufgenommen, die Etherlösung über Magnesiumsulfat getrocknet, eingeengt und der Rückstand fraktioniert destilliert. 1. Fraktion: $1.10 \mathrm{~g} \mathrm{(10 \% )} \mathrm{15g,}{ }^{1} \mathrm{H}-\mathrm{NMR}$-spektroskopisch identisch mit authentischer Substanz ${ }^{49)}$. 2. Fraktion: Gemisch aus $14 \mathrm{~g}$ und $15 \mathrm{~g}$, das mit $90 \mathrm{ml} \mathrm{4proz}$. Salzsäure $14 \mathrm{~h}$ bei $70^{\circ} \mathrm{C}$ gerührt wird. Nach Aufarbeitung wie vorstehend beschricben $2.59 \mathrm{~g}(23 \%)$ 2-Methoxy-1-(methoxyphenylmethyl) benzol $(16 \mathrm{~g})$, Sdp. $120-125^{\circ} \mathrm{C} /$ $2 \cdot 10^{-2}$ Torr. $-{ }^{1} \mathrm{H}-\mathrm{NMR}\left(\mathrm{CDCl}_{3}\right): \delta=3.37\left(\mathrm{~s}, 3 \mathrm{H}, \mathrm{CHOCH}_{3}\right), 3.75\left(\mathrm{~s}, 3 \mathrm{H}, \mathrm{PhOCH}_{3}\right)$, $5.68(\mathrm{~s}, \mathrm{CH}), 6.73-7.63(\mathrm{~m}, 9$ aromat. $\mathrm{H})$.

$$
\mathrm{C}_{15} \mathrm{H}_{16} \mathrm{O}_{2} \text { (228.3) Ber. C } 78.91 \cdot \mathrm{H} 7.06 \text { Gef. C } 79.09 \text { H } 7.04
$$

8. Von 2-(Trimethylsilyl)benzoesäure-ethylester [2-(Ethoxycarbonyl)-1-(trimethylsilyl)benzol] (13h)

13h: Man läßt zu $8 \mathrm{ml}$ Pyridin und $2.5 \mathrm{ml}$ Ethanol unter Eiskühlung und Rühren langsam $5.0 \mathrm{~g} \mathrm{(23.5} \mathrm{mmol)} \mathrm{2-(Chlorcarbonyl)-1} \mathrm{-(trimethylsilyl)benzol}{ }^{50)}$ tropfen, erhitzt anschließend $15 \mathrm{~min}$ auf dem siedenden Wasserbad und gießt nach Abkühlen in $50 \mathrm{ml}$ Eiswasser. Nach Ansäuern mit verd. Salzsäure wird mit Ether ausgeschüttelt, der Etherextrakt über Magnesiumsulfat getrocknet, im Rotationsverdampfer eingeengt und der Rückstand destilliert. Ausb. 2.7 g (52\%) 13h, Sdp. $108-112^{\circ} \mathrm{C} / 13$ Torr. $-{ }^{1} \mathrm{H}-\mathrm{NMR}\left(\mathrm{CDCl}_{3}\right): \delta=0.33$ (s, 9 H, $\left.\mathrm{Si}\left(\mathrm{CH}_{3}\right)_{3}\right), 1.38\left(\mathrm{t}, 3 \mathrm{H}, \mathrm{CH}_{3}\right), 4.35\left(\mathrm{q}, 2 \mathrm{H}, \mathrm{CH}_{2}\right), 7.33-8.13(\mathrm{~m}, 4$ aromat. $\mathrm{H})$.

$$
\mathrm{C}_{12} \mathrm{H}_{18} \mathrm{O}_{2} \mathrm{Si} \text { (222.4) Ber. C } 64.81 \text { H 8.15 Gef. C } 65.00 \text { H } 8.16
$$

a) $0.222 \mathrm{~g}(1.00 \mathrm{mmol}) 13 \mathrm{~h}, 0.212 \mathrm{~g}$ ( $2.00 \mathrm{mmol})$ Benzaldehyd und $0.038 \mathrm{~g}(0.25 \mathrm{mmol})$ Cäsiumfluorid werden in $3 \mathrm{ml}$ HMPT $22 \mathrm{~h}$ bei $100^{\circ} \mathrm{C}$ gerührt. Nach Methode GC/B wird aufgearbeitet. Die gaschromatographische Untersuchung ergibt vollständigen Umsatz zu 3-Phenylphthalid (17).

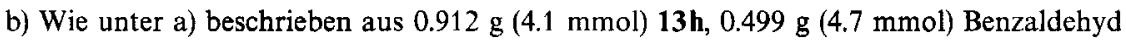
und $0.130 \mathrm{~g}(0.85 \mathrm{mmol})$ Cäsiumfluorid in $4 \mathrm{ml} \mathrm{HMPT}$, jedoch Aufarbeiten nach Methode C. Der erhaltene Rückstand wird in einem Gemisch aus $2 \mathrm{ml}$ 4proz. Salzsäure und $40 \mathrm{ml}$ Methanol $16 \mathrm{~h}$ bei $70^{\circ} \mathrm{C}$ gerührt, das Lösungsmittcl abgezogen, der Rückstand in Ether aufgenommen und die etherische Lösung über Magnesiumsulfat getrocknet. Das Lösungsmittel wird im Rotationsverdampfer entfernt und das zurückbleibende Rohprodukt durch Mitteldrucksäulenchromatographie mit Petrolether/Essigester (10:1) gereinigt. Ausb. $0.57 \mathrm{~g}(66 \%) 17$, Schmp. $114-115^{\circ} \mathrm{C}\left(\right.$ Lit. $\left.^{51)} 116.5-117^{\circ} \mathrm{C}\right)$.

Chem. Ber. 118 (1985) 
9. Von 2-Benzoyl-1-(trimethylsilyl)benzol (13i $)^{41)}: 2.54 \mathrm{~g}(10 \mathrm{mmol}) 13 \mathrm{i}$ werden mit $1.06 \mathrm{~g}$ $(10 \mathrm{mmol})$ Benzaldehyd und $0.29 \mathrm{~g}(5.0 \mathrm{mmol})$ Kaliumfluorid in $30 \mathrm{ml}$ HMPT $24 \mathrm{~h}$ auf $100^{\circ} \mathrm{C}$ erhitzt. Es wird nach Methode $\mathrm{C}$ aufgearbeitet. Der erhaltene Rückstand wird i. Wasserstrahlvak. $4 \mathrm{~h}$ auf $100^{\circ} \mathrm{C}$ erhitzt und der gebildete Feststoff aus Ethanol umkristallisiert. Ausb. $1.67 \mathrm{~g}$ (62\%) 1,3-Diphenylisobenzofuran (18), Schmp. $122-126^{\circ} \mathrm{C}$ (Lit. ${ }^{52}$ $\left.127^{\circ} \mathrm{C}\right)$.

Carbodesilylierung von 2-Nitro-1-(trimethylsilyl)benzol (13a) mit Benzaldehyd in Gegenwart verschiedener Katalysatoren in DMF: Die Suspension des getrockneten (s. vorstehend) und fein verriebenen Katalysators (jeweils $0.10 \mathrm{mmol}=11.2 \mathrm{mg} \mathrm{Me}_{3} \mathrm{COK}, 9.8 \mathrm{mg}$ $\mathrm{MeCO}_{2} \mathrm{~K}, 5.8 \mathrm{mg} \mathrm{KF}, 15.2 \mathrm{mg} \mathrm{CsF}, 18.5 \mathrm{mg} \mathrm{Et}{ }_{4} \mathrm{NF} \cdot 2 \mathrm{H}_{2} \mathrm{O}$ bzw. $30.1 \mathrm{mg} \mathrm{MeCO}_{2} \mathrm{NBu}_{4}$ ) in $3 \mathrm{ml} \mathrm{DMF}$ wird bei Raumtemp. $1 \mathrm{~h}$ gerührt. Anschließend werden bei der angegebenen Temp. die Lösungen von $0.195 \mathrm{~g}(1.0 \mathrm{mmol}) 13 \mathrm{a}$ in $0.5 \mathrm{ml} \mathrm{DMF}$ und $0.106 \mathrm{~g}(1.0 \mathrm{mmol})$ Benzaldehyd in $0.5 \mathrm{ml}$ DMF unter Rühren zugegeben. Bei den Umsetzungen mit $\mathrm{Me}_{3} \mathrm{COK}$ und $\mathrm{Et}_{4} \mathrm{NF} \cdot 2 \mathrm{H}_{2} \mathrm{O}$ erfolgt Selbsterwärmung des Gemisches. Nach der angegebenen Zeit wird nach Methode GC/A, bei der Umsetzung mit CsF nach Methode GC/B aufgearbeitet. Ergebnisse s. Tab. 4.

Carbodesilylierung von 3-Nitro-(13'a) und 4-Nitro-1-(trimethylsilyl)benzol (13'a) mit Benzaldehyd in Gegenwart von $\mathrm{Et}_{4} \mathrm{NF} \cdot 2 \mathrm{H}_{2} \mathrm{O}$ in $\mathrm{HMPT}$

a) Zu der Lösung von $3.91 \mathrm{~g}(20 \mathrm{mmol}) 13 \mathbf{a}^{53)}, 2.12 \mathrm{~g}$ (20 mmol) Benzaldehyd und $25 \mathrm{ml}$ HMPT werden unter Rühren auf einmal $0.93 \mathrm{~g}(5.0 \mathrm{mmol}) \mathrm{Et}_{4} \mathrm{NF} \cdot 2 \mathrm{H}_{2} \mathrm{O}$ gegeben, wobei sich das Gemisch auf ca. $50^{\circ} \mathrm{C}$ erwärmt und rot färbt. Nach $2 \mathrm{~h}$ Rühren wird mit $50 \mathrm{ml}$ 5 proz. Schwefelsäure versetzt, mit Ether extrahiert, die etherische Phase über Magnesiumsulfat getrocknet, im Rotationsverdampfer eingeengt und der Rückstand destilliert. Ausb. $2.79 \mathrm{~g}(61 \%)$ (3-Nitrophenyl) phenylmethanol (15'a), Sdp. $169-171^{\circ} \mathrm{C} / 5 \cdot 10^{-2}$ Torr (Lit. $^{42 \mathrm{~b})}$ $208-212^{\circ} \mathrm{C} / 6$ Torr), Schmp. $68-70^{\circ} \mathrm{C}$ (Lit. ${ }^{42 b)} 68-71^{\circ} \mathrm{C}$ ). ${ }^{1} \mathrm{H}-\mathrm{NMR}$-spektroskopisch identisch mit authentischer Substanz ${ }^{45}$.

b) Wie unter a) beschrieben aus $3.91 \mathrm{~g}$ (20 mmol) $13^{\prime \prime} \mathrm{a}^{53)}, 2.12 \mathrm{~g} \mathrm{(20} \mathrm{mmol)} \mathrm{Benzaldehyd,}$ $25 \mathrm{ml} \mathrm{HMPT}$ und $0.93 \mathrm{~g}(5.0 \mathrm{mmol}) \mathrm{Et}_{4} \mathrm{NF} \cdot 2 \mathrm{H}_{2} \mathrm{O}$; Ausb. $2.47 \mathrm{~g}(54 \%)$ (4-Nitrophenyl)phenylmethanol $\left(\mathbf{1 5}^{\prime \prime} \mathrm{a}\right)$, Sdp. $165-168^{\circ} \mathrm{C} / 5 \cdot 10^{-2}$ Torr, Schmp. $73^{\circ} \mathrm{C}\left(\right.$ Lit. $^{\text {s4) }} 74^{\circ} \mathrm{C}$ ).

Carbodesilylierung von 3-Chlor- (13'b) und 4-Chlor-1-(trimethylsilyl)benzol (13'b) mit Benzaldehyd in Gegenwart von $\mathrm{Me}_{3} \mathrm{COK}$ in $\mathrm{HMPT}$

a) $2.40 \mathrm{~g}$ (13 mmol) $13^{\prime} \mathbf{b}^{55)}, 1.38 \mathrm{~g}(13 \mathrm{mmol})$ Benzaldehyd und $0.437 \mathrm{~g}(3.9 \mathrm{mmol})$ $\mathrm{Me}_{3} \mathrm{COK}$ werden in $50 \mathrm{ml} \mathrm{HMPT} 4 \mathrm{~h}$ bei $100^{\circ} \mathrm{C}$ gerührt, wobei sich das Gemisch grün färbt. Nach Aufarbeiten nach Methode B mit $150 \mathrm{ml} 5$ proz. Schwefelsäure Ausb. $1.45 \mathrm{~g}$ (51\%) (3-Chlorphenyl) phenylmethanol (15'b), Sdp. $134^{\circ} \mathrm{C} / 10^{-2}$ Torr, Schmp. $40^{\circ} \mathrm{C}$ (Lit. ${ }^{56}$ $\left.41^{\circ} \mathrm{C}\right)$.

b) $3.70 \mathrm{~g}(20 \mathrm{mmol}) 1^{\prime \prime} \mathrm{b}^{57)}, 2.12 \mathrm{~g}$ (20 mmol) Benzaldehyd und $0.673 \mathrm{~g}(6.0 \mathrm{mmol}) \mathrm{Me}_{3} \mathrm{COK}$ werden in $50 \mathrm{ml} \mathrm{HMPT} 12 \mathrm{~h}$ bei $100^{\circ} \mathrm{C}$ gerührt. Dann wird nach Methode B aufgearbeitet. Ausb. $2.32 \mathrm{~g} \mathrm{(53 \% )} \mathrm{(4-Chlorphenyl)phenylmethanol}\left(\mathbf{1 5}^{\prime \prime} \mathbf{b}\right), \mathrm{Sdp}, 130^{\circ} \mathrm{C} / 10^{-2}$ Torr, Schmp. $62^{\circ} \mathrm{C}\left(\right.$ Lit. $^{45)} 59-61^{\circ} \mathrm{C}$ ). ${ }^{1} \mathrm{H}$-NMR-spektroskopisch identisch mit authentischer Substanz ${ }^{51)}$.

Vergleichende Umsetzung von 4-Nitro-1-[phenyl(trimethylsiloxy) methyl ]benzol (14'a) mit

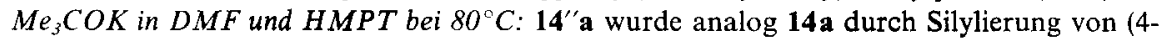
Nitrophenyl)phenylmethanol (15'a) mit Chlortrimethylsilan dargestellt. Jeweils $0.301 \mathrm{~g}(1.00$ mmol) $14^{\prime \prime}$ a und $0.033 \mathrm{~g}(0.30 \mathrm{mmol}) \mathrm{Me}_{3} \mathrm{COK}$ werden in jeweils $3 \mathrm{ml}$ Lösungsmittel bei $80^{\circ} \mathrm{C}$ die angegebene Zeit gerührt. Nach Methode GC/B wird aufgearbeitet. Ausb. in DMF

Chem. Ber. 118 (1985) 
nach 3 h 48\% 1-Benzoyl-4-nitrobenzol (20"a); in HMPT nach 4 h 33\% 20"a und 30\% 14"a; in HMPT nach $12 \mathrm{~h} \mathrm{45 \%} \mathrm{20"a} \mathrm{(jeweils} \mathrm{gaschromatographisch} \mathrm{bestimmt).}$

Carbodesilylierung von 3-Nitro-1-(trimethylsilyl)benzol (13'a) mit Benzaldehyd in Gegenwart von $\mathrm{Me}_{3} \mathrm{COK}$ in DMF bei Raumtemperatur: $\mathrm{Zu} 3.9 \mathrm{~g}(20 \mathrm{mmol}) 1^{\prime} \mathbf{a}^{53)}, 2.12 \mathrm{~g}(20 \mathrm{mmol})$ Benzaldehyd und $25 \mathrm{ml}$ DMF werden unter Rühren bei Raumtemp. in 7 Portionen insgesamt $0.448 \mathrm{~g}$ (4.00 mmol) Me $\mathrm{COK}_{3}$ gegeben. Nach jeweils $1 \mathrm{~h}$ Nachrühren bei Raumtemp. wird eine Probe nach Methode GC/A aufgearbeitet (Tab. 10).

Tab. 10. Produktverteilung bei der Carbodesilylierung von 3-Nitro-1-(trimethylsilyl)benzol (13'a) mit Benzaldehyd unter portionsweiser Zugabe von insgesamt $20 \mathrm{~mol}-\% \mathrm{Me}_{3} \mathrm{COK}$

\begin{tabular}{|c|c|c|c|c|c|c|c|c|}
\hline \multirow{2}{*}{ Produkte } & \multicolumn{7}{|c|}{ Ausb. $\%{ }^{a}$ nach der } & \multirow{2}{*}{$\begin{array}{l}\text { Zugabe ar } \\
\mathrm{Me}_{3} \mathrm{COK}\end{array}$} \\
\hline & 1. & 2. & 3. & 4. & 5. & 6. & 7. & \\
\hline $13 ' a$ & 100 & 89 & 84 & 77 & 52 & 32 & 14 & \\
\hline $\begin{array}{l}\text { 3-Nitro-1-[phenyl- } \\
\text { (trimethylsiloxy)- } \\
\text { methyl] benzol } \\
\text { (14'a) }\end{array}$ & - & 11 & 13 & 20 & 41 & 24 & 11 & \\
\hline $\begin{array}{l}\text { (3-Nitrophenyl)- } \\
\text { phenylmethanol } \\
\left(\mathbf{1 5}^{\prime} \mathbf{a}\right)\end{array}$ & - & - & 2 & 2 & 6 & 27 & 38 & \\
\hline $\begin{array}{l}\text { 1-Benzoyl-3- } \\
\text { nitrobenzol } \\
\left(20^{\prime} \mathbf{a}\right)\end{array}$ & - & - & - & - & - & 11 & 21 & \\
\hline Nitrobenzol & - & - & 1 & 1 & 1 & 6 & 16 & \\
\hline
\end{tabular}

a) Gaschromatographisch bestimmt.

Nach 14tägigem Stehenlassen bei Raumtemp. wird das Reaktionsgemisch in $75 \mathrm{ml} \mathrm{Was-}$ ser eingerührt. Dann wird mit Chloroform extrahiert, der Chloroformextrakt über Magnesiumsulfat getrocknet, das Chloroform im Rotationsverdampfer entfernt und der Rückstand destilliert. Ausb. $0.91 \mathrm{~g} \mathrm{(20 \% )} \mathrm{1-Benzoyl-3-nitrobenzol} \mathrm{(20'a),} \mathrm{Sdp.} \mathrm{136-138}{ }^{\circ} \mathrm{C} / 5 \cdot 10^{-3}$ Torr (Lit. ${ }^{581} 234^{\circ} \mathrm{C} / 18$ Torr), Schmp. $93-95^{\circ} \mathrm{C}$ ( Lit. $^{58)} 94-95^{\circ} \mathrm{C}$ ). ${ }^{1} \mathrm{H}-\mathrm{NMR}$-spektroskopisch identisch mit authentischer Substanz ${ }^{58)}$.

Umsetzung von 2-Nitro-1-[phenyl(trimethylsiloxy)methyl]benzol (14a) mit 33 mol-\% $\mathrm{Me}_{3} \mathrm{COK}$ in DMF: $0.15 \mathrm{~g}(0.50 \mathrm{mmol}) 14 \mathrm{a}$ werden mit $0.016 \mathrm{~g}(0.15 \mathrm{mmol}) \mathrm{Me}_{3} \mathrm{COK}$ in $4 \mathrm{ml} \mathrm{DMF} 8 \mathrm{~h}$ bei $100^{\circ} \mathrm{C}$ gerührt. Es wird nach Methode GC/B aufgearbeitet. Ausb. $27 \%$ 1-Benzoyl-2-nitrobenzol (20a). 14a und (2-Nitrophenyl)phenylmethanol (15a) konnten nicht nachgewiesen werden.

Versuche zur Carbodesilylierung von (Trimethylsilyl)benzol: $\mathrm{Zu} 3.0 \mathrm{~g}(20 \mathrm{mmol})$ (Trimethylsilyl)benzol ${ }^{43)}$ und $2.12 \mathrm{~g}(20 \mathrm{mmol})$ Benzaldehyd in $50 \mathrm{ml}$ HMPT werden portionsweise unter Rühren $1.57 \mathrm{~g}(14 \mathrm{mmol}) \mathrm{Me}_{3} \mathrm{COK}$ gegeben, anschließend wird nach Methode GC/B aufgearbeitet. Durch Koinjektion wird Benzhydrol (in verschiedenen Ansätzen 5, 15 bzw. 22\%) gaschromatographisch nachgewiesen, außerdem verschiedene Mengen Benzoesäure-benzylester.

Carbodesilylierung von 2-(Trimethylsilyl) thiophen (21 $)^{43)}: \mathrm{Zu} 7.8 \mathrm{~g}(50 \mathrm{mmol}) 21$ und $5.30 \mathrm{~g}$ (50 mmol) Benzaldehyd in $50 \mathrm{ml}$ DMF werden bei Raumtemp. auf einmal $0.112 \mathrm{~g} \mathrm{(1.00}$

Chem. Ber. 118 (1985) 


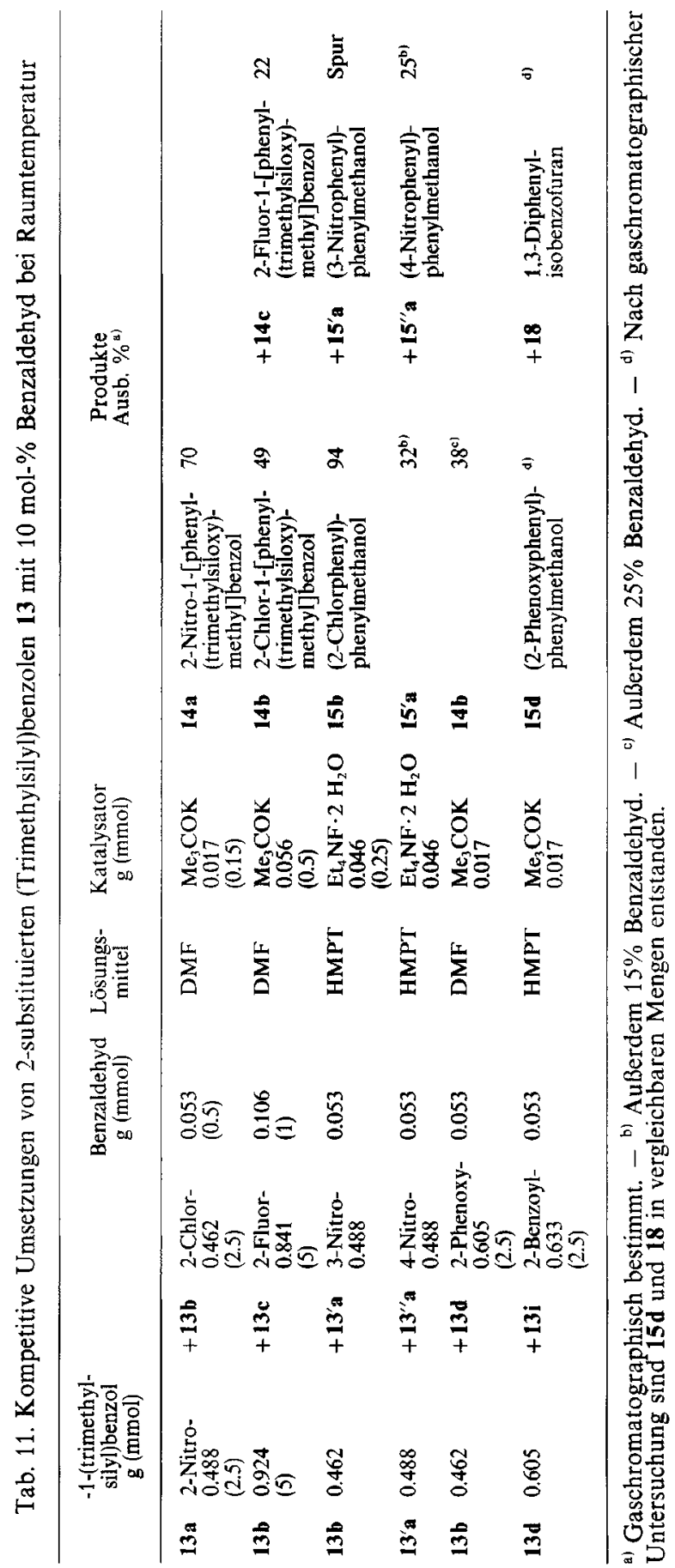

Chem. Ber. $1 / 8$ (1985) 
mmol) $\mathrm{Me}_{3} \mathrm{COK}$ gegeben, wobei sich das Gemisch auf $70^{\circ} \mathrm{C}$ erwärmt und rot färbt. Nach $1 \mathrm{~h}$ Rühren wird nach Methode A aufgearbeitet, Ausb. $10.50 \mathrm{~g}(80 \%) 2-[$ Phenyl (trimethylsiloxy)methyl thiophen (22), Sdp. $152^{\circ} \mathrm{C} / 14$ Torr. - ${ }^{1} \mathrm{H}-\mathrm{NMR}\left(\mathrm{CDCl}_{3}\right): \delta=0.10(\mathrm{~s}, 9 \mathrm{H}$, $\left.\mathrm{Si}\left(\mathrm{CH}_{3}\right)_{3}\right), 6.01(\mathrm{~s}, \mathrm{CH}), 6.73-7.00(\mathrm{~m}, 2 \mathrm{H}$, Thiophen-3,4-H), $7.13-7.60(\mathrm{~m}, 6 \mathrm{H}$, Thiophen5-H, Ph).

\section{$\mathrm{C}_{14} \mathrm{H}_{18}$ OSSi (262.4) $\quad$ Ber. C 64.07 H 6.91 S 12.21 Gef. C 64.30 H 6.96 S 12.17}

22 zersetzt sich bei Raumtemp. innerhalb 14 Tagen unter Bildung von zwei Phasen, von denen die spezifisch leichtere klare Phase gaschromatographisch und ${ }^{1} \mathrm{H}-\mathrm{NMR}$-spektroskopisch als Hexamethyldisiloxan identifiziert wurde.

Carbodesilylierung von 3-(Trimethylsilyl)thiophen $\left(21^{\prime}\right)^{43)}: 10.94 \mathrm{~g}(70 \mathrm{mmol}) \mathbf{2 1}^{\prime}$ werden mit $7.42 \mathrm{~g}(70 \mathrm{mmol})$ Benzaldehyd und $1.40 \mathrm{~g}$ (9.2 mmol) CsF in $70 \mathrm{ml}$ HMPT $24 \mathrm{~h}$ bei $100^{\circ} \mathrm{C}$ gerührt. Anschließend wird nach Methode C (mit $250 \mathrm{ml}$ Eismischung) schnell aufgearbeitet. Der Rückstand wird destilliert. Ausb. $7.71 \mathrm{~g} \mathrm{(42 \% )} \mathrm{3-/Phenyl} \mathrm{(trimethylsiloxy).}$ methyl Jithiophen (22'), Sdp. $150-154^{\circ} \mathrm{C} / 12$ Torr, Schmp. $19-21^{\circ} \mathrm{C} .-{ }^{1} \mathrm{H}-\mathrm{NMR}\left(\mathrm{CDCl}_{3}\right)$ : $\delta=0.07\left(\mathrm{~s}, 9 \mathrm{H}, \mathrm{Si}\left(\mathrm{CH}_{3}\right)_{3}\right), 5.83(\mathrm{~s}, \mathrm{CH}), 6.86-7.01(\mathrm{~m}, 1 \mathrm{H}$, Thiophen-4-H), $7.01-7.50(\mathrm{~m}$, $7 \mathrm{H}$, Thiophen-2,5-H, Ph).

\section{$\mathrm{C}_{14} \mathrm{H}_{18} \mathrm{OSSi}(262.4)$ Ber. C 64.07 H 6.91 S 12.21 Gef. C 64.22 H 6.96 S 12.05}

Kompetitive Umsetzungen von 2-substituierten (Trimethylsilyl)benzolen 13 mit Benzaldehyd bei Raumtemperatur: Dic Edukte werden mit Benzaldehyd und dem Katalysator bei Raumtemp. in ca. $5 \mathrm{ml}$ DMF oder HMPT gerührt und nach Beendigung der Reaktion des Benzaldehyds nach Methode GC/A (beim Arbeiten in DMF) bzw. GC/B (beim Arbeiten in HMPT) aufgearbeitet (Tab. 11).

${ }^{1}$ Zur elektrophilen Aromatensubstitution, 27; 26. Mitteil.: E. Effenberger und A. Krebs, J. Org. Chem. 49, 4687 (1984).

2) W. Spiegler. Teil der Dissertation, Univ. Stuttgart 1980.

3) 3a) C. Eaborn, J. Organomet. Chem. 100, 43 (1975), Zusammenfassung mit weiteren Literaturhinweisen. - ${ }^{3 b)}$ I. Szele, Helv. Chim. Acta 64, 2733 (1981).

4) 4a) C. L. Perrin, J. Org. Chem. 36, 420 (1971). - 4b) S. R. Hartshorn, Chem. Soc. Rev. 3, 167 (1974).

5) Sa) G. Félix, J. Dunoguès und $R$. Calas, Angew. Chem. 91, 430 (1979); Angew, Chem., Int. Ed. Engl. 18, 402 (1979). - ${ }^{5 b)} J$. P. Dunoguès, Chemtech 1982, 373, dort weitere Literaturhinweise. - ${ }^{\text {c) }}$ R. L. Funk und K.P.C. Vollhardt, J. Am. Chem. Soc. 99, 5483 (1977). - ${ }^{\text {dd }}$ R. L. Funk und K. P. C. Vollhardt, J. Am. Chem. Soc. 101, 215 (1979). ${ }^{\text {sel }}$ D. S. Wilbur, W. E. Stone und K. W. Anderson, J. Org. Chem. 48, 1542 (1983).

6) 6a) W.P. Weber, Silicon Reagents for Organic Synthesis, Reactive and Structure Concepts in Organic Chemistry, Vol. 14, Springer Verlag, Berlin, Heidelberg, New York 1983. 6b) E. W. Colvin, Silicon in Organic Synthesis, Butterworths Monographs in Chemistry and Chemical Engineering, Butterworth, London 1981 - ${ }^{6 c)}$ A. Ricci, A. Degf innocenti, M. Fiorenza, M. Taddei, M. A. Spartera und D. R. M. Walton, Tetrahedron Lett. 23, 577 (1982).

n) †н) O. A. Reutov, I. P. Beletskaya und K. P. Butin, CH-Acids, S. $85 \mathrm{ff.}$., Pergamon Press, Oxford 1978. - ${ }^{76)}$ R. R. Fraser, M. Bresse und T. S. Mansour, J. Am. Chem. Soc. 105, $7790(1983)$.

8) 8a) C. Eaborn, Intra Sci. Chem. Rep. 7, 97 (1975). - ${ }^{\text {8b }}$ C. Eaborn, J. G. Stamper und G. Seconi, J. Organomet. Chem. 204, 27 (1981). - ${ }^{8 c)}$ G. Seconi, M. Taddei, C. Eaborn und J. G. Stamper, J. Chem. Soc., Perkin Trans. 2 1982, 643. - ${ }^{8 d)}$ J. Cretney und G. J. Wright, J. Organomet. Chem. 28, 49 (1971).

9) $F$. Effenberger und $W$. Spiegler, Angew. Chem. 93, 287 (1981); Angew. Chem., Int. Ed. Engl. 20, 265 (1981).

10) A. F. Webb, D. S. Sethi und H. Gilman, J. Organomet. Chem. 21, P 61 (1970).

11) 11a) $N$. Ishikawa und $K$. Isobe, Chem. Lett. 1972, 435. - ${ }^{11 b) ~ B . ~ A . ~ G o s t e v s k i i, ~ O . ~ A . ~}$ Krualava, A. I. Albanov und N. S. Vyazankin, J. Organomet. Chem. 187, 157 (1980). 
12) O. $\breve{A}$. Reutov, Tetrahedron 34, 2827 (1978).

13) E. A. V. Ebsworth in A. G. MacDiarmid, Organometallic Compounds of the Group IV Elements, Vol. 1, S. 45, M. Dekker Inc., New York 1968.

14) 14a) F. H. Pinkerton und S. F. Thames, J. Heterocycl. Chem. 8, $257(1971) .-{ }^{14 b)} F . H$. Pinkerton und S. F. Thames, J. Heterocycl. Chem. 9, 67 (1972).

${ }^{15)}{ }^{15 a)} A$. Streitwieser jr. und P. J. Scannon, J. Am. Chem. Soc. 95, 6273 (1973). $-{ }^{15 b)} R . V$. Kendall, Dissertation, Pennsylvania State Univ. 1970; Diss. Abstr. Int. B 1971, 32 (1) 172 [Chem. Abstr. 76, 3267x (1972)].

16) (6a) L. Birkofer, A. Ritter und H. Weiden, Chem. Ber. 95, 971 (1962). - ${ }^{16 b)}$ A. Hosomi, A. Shirahata und $H$. Sakurai, Tetrahedron Lett. 1978, 3043.

${ }^{17)}$ M. Deneux, I. C. Akhrem, D. V. Avetissian, E. I. Myssoff und M. E. Volpin, Bull. Soc. Chim. Fr. 1973, 2638.

${ }^{18)}$ R. C. Weast. Handbook of Chemistry and Physics, 63. Ed., D-171/172, The Chemical Rubber Co., Cleveland, Ohio 1982-1983.

19) W. K. McEwen. J. Am. Chem. Soc. 58, 1124 (1936).

${ }^{20)} R$. K. Sharma und J. L. Fry, J. Org. Chem. 48, 2112 (1983).

${ }^{21)}$ G. G. Jakobson und N. E. Akhmetova, Synthesis 1983, 169.

22) 22a) R. G. Pearson, J. Am. Chem. Soc. 85, $3533(1963)$. - ${ }^{22 b)}$ R. G. Pearson und J. Songstad, J. Am. Chem. Soc. 89, 1827 (1967). - ${ }^{22 c)}$ Tse-Lok Ho, Hard and Soft Acids and Bases Principle in Organic Chemistry, S. 167, Academic Press, New York, San Francisco, London 1977.

23) R. J. P. Corriu und C. Guérin, Tetrahedron 37, 2467 (1981).

24) R. J. P. Corriu und C. Guérin, Adv. Organomet. Chem. 20, 265 (1982).

${ }^{25)}$ R. R. Holmes, R. O. Day, J. J. Harland, A. C. Sau und J. M. Holmes, Organometallics 3, 341 (1984).

26) 26a) $P$. Jutzi und $O$. Lorey, J. Organomet. Chem. 104, 153 (1971). - ${ }^{26 \mathrm{~b})} P$. Jutzi und H.-J. Hoffmann, Chem. Ber. 106, 594 (1973). - ${ }^{26 c)} J$. A. Zoltewicz und L. W. Deady, Adv. Heterocycl. Chem. 22, 71 (1978).

27) M. M. A. Guyot und I. Catel, Bull. Soc. Chim. Fr. 35, 1124 (1906) [Chem. Abstr. 1, 853 (1907)].

28) A. I. Shatenstein, A. G. Kamrad, I. O. Shapiro, Y. I. Ranneva und E. N. Zvyaqintseva, Khim. Seraorg. Soedin 1972, 121 [Chem. Abstr. 80, 59256t (1974)].

${ }^{29)} J$. F. Bunnett in Techniques of Organic Chemistry, 3. Aufl., Bd. VI, S. 158, Wiley-Interscience Publication, New York 1974.

30) G. B. Barlin und D. D. Perrin, Quart. Rev. (London) 20, 75 (1966).

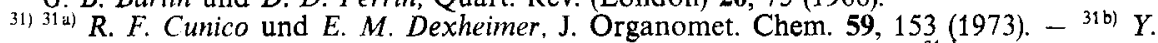
Himeshima, T. Sonoda und H. Kobayashi, Chem. Lett. 1983,1211. - ${ }^{310)} J . R$. Mills und $V$. Snieckus, Tetrahedron Lett. 25, 483 (1984).

32) Vorgesehene Dissertation W. Daub, Univ. Stuttgart.

33) R. Kaiser, Chromatographie in der Gasphase, S. 209, Bibliographisches Institut, Mannheim 1965.

${ }^{34)}$ R. Glatz, Dissertation, Univ. Stuttgart 1977.

${ }^{35)}$ A. J. Speziale, K. W. Ratts und D. E. Bissing, Org. Synth., Coll. Vol. V, 361 (1973).

${ }^{36)}$ H. Kobler, R. Munz, G. Al Gasser und G. Simchen, Liebigs Ann. Chem. 1978, 1937.

37) I. N. Rozhkov und I. L. Knunyants, Dokl. Akad. Nauk SSSR 1971, 199 B, 614 [Chem. Abstr. 76, 7291 y (1972)].

${ }^{38}$ W. Lang, Ber. Dtsch. Chem. Ges. 25, 1902 (1892).

39) P. Jutzi, H.-J. Hoffmann, K. Beier und K. H. Wyes, J. Organomet. Chem. 82, 209 (1974).

40) I. Haiduc und H. Gilman, Rev. Roum. Chim. 1971, 16, 305 [Chem. Abstr. 75, 36230n (1971)].

41) C. Eaborn, D. R. M. Walton und D. J. Young, J. Chem. Soc. B 1969, 15.

42) 42a) D. H. Hey und R. D. Mulley, J. Chem. Soc. 1952, 2283. - ${ }^{42 b)} M$. S. Newman und $A$. S. Smith. J. Org. Chem. 13, 592 (1948).

${ }^{431}$ F. Effenberger und D. Häbich, Liebigs Ann. Chem. 1979, 842.

${ }^{44}$ C. Eaborn, K. L. Jaura und D. R. M. Walton, J. Chem. Soc. 1964, 1198.

45) W. C. Bachmann, E. Carlson jr. und J. C. Moran, J. Org. Chem. 13, 916 (1948).

46) W. Fink, Helv. Chim. Acta 56, 355 (1973).

47) 47 a) U. Schöllkopf in Methoden der organischen Chemie (Houben-Weyl-Müller), 1. Aufl., Bd. 13/1, S. 87, Thieme, Stuttgart 1970. - ${ }^{47 b)}$ G. Köbrich, Chem. Ber. 92, 2981 (1959).

${ }^{48}$ M. S. A. Koopal, Rec. Trav. Chim. Pays-Bas 34, 162 (1915).

${ }^{49)}$ R. Stoermer und E. Frederici, Ber. Dtsch. Chem. Ges. 41, 332 (1908). 
${ }^{50)}$ R. A. Benkeser und H. R. Krysiak, J. Am. Chem. Soc. 76, 599 (1954).

${ }^{51)} D$. Y. Curtin und H. J. Fletcher, J. Org. Chem. 19, 352 (1954).

${ }^{52)}$ C. F. H. Allen und J. A. VanAllan, J. Am. Chem. Soc. 70, 2069 (1948).

53) J. L. Speier, J. Am. Chem. Soc. 75, 2930 (1953).

${ }^{54)} H$. Lund, Ber. Dtsch. Chem. Ges. 70, 1520 (1937).

${ }^{55)}$ H. A. Clark, A. F. Gordon, C. W. Young und M. J. Hunter, J. Am. Chem. Soc. 73, 3798 (1951).

56) L. C. Bateman, E. D. Hughes und C. K. Ingold, J. Chem. Soc. 1940, 1011.

57) C. A. Burkhard, J. Am. Chem. Soc. 68, 2103 (1946).

${ }^{58)} K$. Ziegler und F. Dithel, Liebigs Ann. Chem. 473, 194 (1929). 